\title{
ORBITAL CONFIGURATIONS AND DYNAMICAL STABILITY OF MULTIPLANET SYSTEMS AROUND SUN-LIKE STARS HD 202206, 14 HERCULIS, HD 37124, AND HD 108874
}

\author{
Krzysztof Goździewski, ${ }^{1}$ Maciej Konacki, ${ }^{2}$ And Andrzej J. Maciejewski ${ }^{3}$ \\ Received 2005 November 15; accepted 2006 February 23
}

\begin{abstract}
We perform a dynamical analysis of the recently published radial velocity (RV) measurements of a few solar-type stars that host multiple Jupiter-like planets. In particular, we reanalyze the data for HD 202206, 14 Her, HD 37124, and HD 108874. We derive dynamically stable configurations that reproduce the observed RV signals, using GAMP (the genetic algorithm with MEGNO penalty). GAMP relies on $N$-body dynamics and makes use of genetic algorithms merged with a stability criterion. For this purpose, we use the maximal Lyapunov exponent computed with the dynamical fast indicator MEGNO. Through a dynamical analysis of the phase space in a neighborhood of the obtained best-fit solutions, we derive meaningful limits on the parameters of the planets. We demonstrate that GAMP is especially well suited to the analysis of the RV data that only partially cover the longest orbital period and/or are related to multiplanet configurations involved in low-order mean motion resonances (MMRs). Our analysis reveals a presence of a second Jupiter-like planet in the 14 Her system $(14 \mathrm{Her}$ c) that is involved in a $3: 1$ or $6: 1$ MMR with the known companion $14 \mathrm{Her}$ b. We also show that the dynamics of the HD 202206 system may be qualitatively different when coplanar and mutually inclined orbits of the companions are considered. We demonstrate that the two outer planets in the HD 37124 system may reside in a close neighborhood of the $5: 2 \mathrm{MMR}$. Our results confirm that the HD 108874 system may be very close to a, or locked in an exact, $4: 1$ MMR.
\end{abstract}

Subject headings: celestial mechanics — methods: $n$-body simulations - methods: numerical — planetary systems stars: individual (HD 202206, 14 Herculis, HD 37124, HD 108874) — stellar dynamics

Online material: color figures

\section{INTRODUCTION}

Finding the best-fit solutions to radial velocity (RV) observations of stars with more than one planet that only partially cover the longest orbital period is difficult. In such a case, a kinematic superposition of the Keplerian orbits, or even the $\mathrm{N}$-body model of the RV, may lead to configurations with a poorly constrained eccentricity of the outermost planet (Jones et al. 2002; Goździewski et al. 2003). In the statistically optimal best-fit solutions, the eccentricities can be large and can quickly (on the timescale of thousands of years) lead to catastrophic collisional instability. Moreover, the validity of a superposition of kinematic Keplerian signals can be very problematic for systems that are involved in low-order mean motion resonances (MMRs). Due to significant uncertainties and the small number of the measurements, even the $\mathrm{N}$-body model of the RV curve that incorporates the mutual gravitational interactions frequently yields unstable configurations because the model is "blind" to the sophisticated, fractal-like structure of the orbital parameter space as predicted by the fundamental Kolmogorov-Arnold-Moser theorem (Arnold 1978). According to this theorem, the phase space of a planetary system is discontinuous with respect to the requirement of stability.

An ideal fitting algorithm should find a solution that reproduces the RV data and simultaneously corresponds to a stable planetary configuration. The most frequently used notion of the

\footnotetext{
1 Torun Centre for Astronomy, Nicolaus Copernicus University, Gagarina 11, 87-100 Toruń, Poland; k.gozdziewski@astri.uni.torun.pl.

${ }_{2}$ Nicolaus Copernicus Astronomical Center, Polish Academy of Sciences, Rabiańska 8, 87-100 Toruń, Poland; and Division of Geological and Planetary Sciences, California Institute of Technology, MS 150-21, 1201 East California Boulevard, Pasadena, CA 91125; maciej@ncac.torun.pl.

${ }^{3}$ Institute of Astronomy, University of Zielona Góra, Podgórna 50, 65-246 Zielona Góra, Poland; maciejka@astro.ia.uz.zgora.pl.
}

term "stable" means "not disrupting or qualitatively changing during relatively short periods of time," say, millions of years. This idea has been already used by many authors in modeling the $\mathrm{RV}$ data. We used it for a dynamical confirmation of the $2: 1$ MMR in the HD 82943 system (Goździewski \& Maciejewski 2001). Recently, Ferraz-Mello et al. (2005), Correia et al. (2005), and Vogt et al. (2005) have applied such an approach in the analysis of the RV data of multiplanet systems (in particular, of hypothetically resonant configurations).

Often, a stability criterion is applied after the mathematically best-fit (but unstable) solution is found, and the orbital elements are then adjusted to obtain a stable configuration. We also show that such a modification of the best-fit initial condition does not necessarily give an optimal stable solution. In our relatively new method, the Genetic Algorithm with MEGNO Penalty (GAMP), the stability analysis is an internal part of the fitting procedure (Goździewski et al. 2003, 2005). We treat the dynamical behavior in terms of the chaotic and regular (or weakly chaotic) states as an additional observable that is weighted at the same level of importance as the RV measurements. We penalize the strongly unstable solutions by artificially increasing their $\left(\chi_{\nu}^{2}\right)^{1 / 2}$. When determining the character of motions, we rely on the computation of the maximal Lyapunov exponent through the mean exponential growth factor of nearby orbits (MEGNO) indicator (Cincotta \& Simó 2000; Cincotta et al. 2003; Giordano \& Cincotta 2004). Apparently the use of such a formal criterion of the stability for modeling real data may be problematic. Almost any planetary system, including our own, can be very close to a chaotic state. Nevertheless, we expect that even if chaos appears, it should not impair the astronomical stability (Lissauer 1999), by which we mean that planetary orbits are bounded over a very long time and that no collisions or ejection of planets occur. For configurations involving Jupiter-like companions in close orbits with (typically) 
large eccentricities, the formal stability seems to be closely related to the astronomical stability. A serious complication is that there is no known general relation between the Lyapunov time (a characteristic time of the formal instability) and the event time; that is, the time after which a physically significant change of the planetary configuration happens (see, e.g., Lecar et al. 2001; Michtchenko \& Ferraz-Mello 2001). Still, the chaotic motions may easily destabilize the planetary configuration over a short timescale related to the relevant low-order MMRs. It can be well explained for the twoplanet configurations close to coplanar systems. By applying the recent secular theories of Michtchenko \& Malhotra (2004) and Lee \& Peale (2003), one can predict that the main sources of short-time instabilities are related to low-order MMRs or to the proximity of the system to collision zones. Outside the resonances and far from the collision zones, the planetary system is generically stable, even in the range of large eccentricities. These works generalize the Laplace-Lagrange secular theory (Murray $\&$ Dermott 2000). For the $N$-planet system, Pauwels (1983) derived a similar conclusion, which is nevertheless formally restricted to the range of small eccentricities. In the neighborhood of the collision zone, the MMRs overlap, and that leads to the origin of a region of global instability. The fitting process should certainly eliminate initial conditions in such zones, and, in general, eliminate strongly chaotic motions related to unstable regions of the MMRs. Fortunately, these can be detected numerically, thanks to efficient fast indicators over the characteristic event timescale, which is measured roughly in units of $10^{4}-10^{5}$ times the longest orbital periods.

In this paper, we reanalyze the RV data for HD 202206 (Correia et al. 2005), 14 Her (Naef et al. 2004), and HD 37124 and HD 108874 (Vogt et al. 2005), using GAMP. A common feature of these systems is that the available measurements either only partially cover, or cover a small number of, the longest orbital periods. The planetary systems reside in the zones spanned by strong loworder MMRs. This work extends the results of our recent papers, which were devoted to $\mu$ Arae (Goździewski 2003; Goździewski et al. 2003) and HD 82943 and HD 123811 (Goździewski \& Konacki 2006). The studied systems are selected as representative cases found among the detected multiplanet configurations.

\section{THE NUMERICAL SETUP AND THE FITTING METHOD}

In order to incorporate the theoretical ideas described in the previous section, we employ a few numerical tools and merge the results in a self-consistent manner. To efficiently explore the phase space (whose structure is understood in terms of the Kolmogorov-Arnold-Moser [KAM] theorem), we use the genetic algorithm (GA) scheme implemented by Charbonneau (1995). The GAs make it possible, in principle, to find the global minimum of $\left(\chi_{\nu}^{2}\right)^{1 / 2}$. In the GAMP code, the solutions to which the GAs converged are finally refined by a very accurate nongradient simplex scheme through the Nelder-Mead method (Press et al. 1992). The fractional convergence tolerance to be achieved in the simplex code is set in the range $10^{-4}-10^{-6}$ (typically, the lower accuracy is forced in time-consuming GAMP tests). The simplex refinement in the CPU-expensive GAMP code reduces the CPU usage dramatically by a factor of tens. ${ }^{4}$ Yet a single particular initial condition may be fine-tuned to match the accuracy required (or that it is possible to obtain).

The reflex motion of a star is described with the self-consistent Newtonian $N$-body model (Laughlin \& Chambers 2001). The

\footnotetext{
4 The fitting code can then be called GAMPS (Genetic Algorithm with MEGNO Penalty and Simplex).
}

character of planetary motions is determined in terms of the Lyapunov characteristic exponent, which is expressed by the MEGNO indicator (Cincotta et al. 2003). Thanks to the excellent sensitivity of this indicator to chaotic motions (particularly when accompanying close encounters), very short integration times are sufficient to remove the most unstable initial conditions. Typically, the computations rely on $\sim 10^{3}-10^{4}$ orbital periods of an outermost body. This is not long enough to eliminate all chaotic motions, but we are left (and in fact it is a desired feature of the method) with regular or weakly chaotic configurations that are typically located on the borders of stable zones. We note that the GAMP code may use basically any arbitrary stability criterion. In this sense, the method is quite general. The definition of the KAM stability, which is directly related to the Lyapunov exponent, seems to be the most natural and well justified by the theoretical considerations.

The dynamical neighborhood of a best-fit solution is examined by other fast indicators. The first one derived on the basis of the fast Fourier transform (FFT) is called the spectral method (SM; Michtchenko \& Ferraz-Mello 2001). A refined and more complex method of this type is the frequency analysis by Laskar (1993). In our work, the SM is employed to resolve the structure of the spectral signal produced by short-term dynamics (i.e., the proper mean motion as one of the fundamental frequencies). After many comparative tests, we found that both MEGNO and the SM are similarly sensitive to the chaotic diffusion generated by the MMRs in systems with Jupiter-like planets. To detect it, the required integration time is relatively very short, typically about $10^{4}$ orbital periods of the outermost planet. Under some conditions, the SM is even more efficient than MEGNO, because one avoids the integration of complex variational equations. It also provides a straightforward identification of the MMRs. The SM is used for computations of dynamical maps in two-dimensional planes of selected osculating elements. Yet another fast indicator that helps us to detect physically significant changes of the orbital configurations is the max $e$ indicator (the maximal eccentricity attained by the orbit of the investigated planet during a prescribed integration time). We use all three fast indicators, as they complement each other. This makes it possible to examine the dynamical properties of the best-fit solutions through different characteristics of the dynamics: the maximal Lyapunov exponent, the variation of the fundamental frequencies, and the geometrical evolution of orbits.

\section{A PLANETARY SYSTEM IN AN EXACT MMR (HD 202206)}

The two-planet system around HD 202206 was discovered by the Geneva Extrasolar Planet Search team (Correia et al. 2005). In this system a massive Jupiter-like planet or a brown dwarf is accompanied by a smaller Jovian body in a more distant orbit. The analysis conducted by Correia et al. (2005) revealed that both planets are likely involved in a 5:1 MMR. They found that both the best double-Keplerian and $N$-body solutions were very unstable and led to a disintegration of the system during a few thousand years. In order to find a dynamically stable solution, the authors have computed the two-dimensional stability map (in terms of the diffusion rate of the proper mean motion) in the neighborhood of the best Newtonian fit. Next, on the basis of this map, they have selected a stable configuration by a rather arbitrary postfit adjusting of the orbital parameters. Certainly, we should not expect that such changes of the mathematically best-fit initial condition will provide a statistically optimal fit to the data.

The RV data of HD 202206 could be used to test our approach and to confirm the results of the dynamical analysis performed by the discovery team. Unfortunately, the full set of RVs has not 

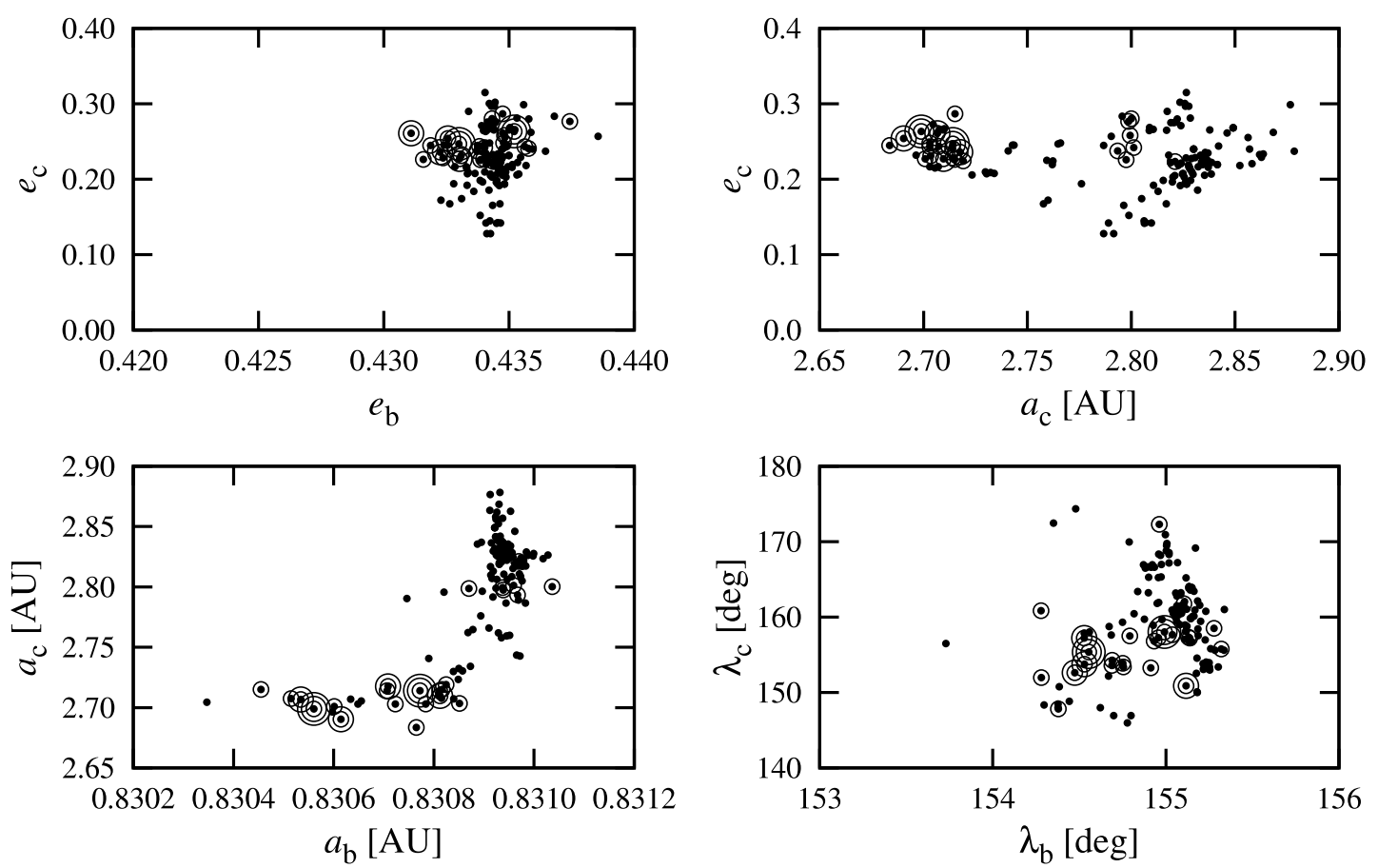

Fig. 1.-Best fits obtained by the GAMP algorithm for the RV data published graphically in Correia et al. (2005) for HD 202206. The system is assumed to be coplanar. Parameters of the fit are projected onto the planes of osculating orbital elements at the epoch of the first observation, JD 2,451,402.8027. The smallest filled circles indicate stable solutions with $\left(\chi_{\nu}^{2}\right)^{1 / 2}<1.65$ and an $\mathrm{rms}$ of $\sim 11 \mathrm{~m} \mathrm{~s}^{-1}$. Larger open circles indicate $\left(\chi_{\nu}^{2}\right)^{1 / 2}<1.55$ and $\left(\chi_{\nu}^{2}\right)^{1 / 2}<1.6[$ the formal $1 \sigma$ confidence interval of the best-fit solution is $\left.\left(\chi_{\nu}^{2}\right)^{1 / 2}=1.53\right]$. The largest circles indicate the solutions with $\left(\chi_{\nu}^{2}\right)^{1 / 2}<1.52$, which is marginally larger than the value of $\left(\chi_{\nu}^{2}\right)^{1 / 2}=1.519$ in the best-fit initial condition (Table 1).

been published. In order to overcome this problem, we extracted the data from the relevant figure from Correia et al. (2005). A large fraction of the measurements has been published in an earlier paper (Udry et al. 2002). This set consists of 95 measurements. Some of them (apparently the most uncertain ones) have been removed and do not appear in the data set used by Correia et al. (2005), which consists of 105 data points. By comparing the common part of the data sets, we can precisely estimate the quality of the scanned "measurements." We found that the standard deviation of the differences between the relevant data points is less than $1 \mathrm{~m} \mathrm{~s}^{-1}$ in RVs and $\sim 0.3$ days in the moments of observations. These "errors" are very small when compared to large variations of the RV signal $\left(\sim 1200 \mathrm{~m} \mathrm{~s}^{-1}\right)$ and long orbital periods. Indeed, using the synthetic data, we can recover the solutions by Correia et al. (2005).

The results of the GAMP analysis are illustrated in Figure 1. We ran the code a few hundred times and collected the solutions for which the procedure converged. The parameters of these solutions are illustrated by projections onto the representative planes of the osculating elements at the initial epoch of the first observation (note that to directly compare our results with those obtained by the discovery team, the RV measurements are not rescaled by the stellar jitter). In quite an extensive search, we looked only for coplanar configurations. In Figure 1 we mark only stable solutions with $\left(\chi_{\nu}^{2}\right)^{1 / 2}<1.65$ (small filled circles). That value of $\left(\chi_{\nu}^{2}\right)^{1 / 2}$ is comparable with the value of $\left(\chi_{\nu}^{2}\right)^{1 / 2}$ of the best stable fit S5 from Correia et al. (2005). The application of GAMP makes it possible to find a better solution, with $\left(\chi_{\nu}^{2}\right)^{1 / 2}=1.52$ and an rms of $\sim 10 \mathrm{~m} \mathrm{~s}^{-1}$. The stability analysis of this fit is illustrated in the left panels of Figure 2. The solution can be found close to the border of a relatively narrow stable island of the $5: 1$ MMR (Fig. 2, top left, showing $\log \mathrm{SN}$ ). In the same integration we computed the indicator max $e_{c}$ (Fig. 2, bottom left). The al- most perfect coincidence of these two plots is striking. It means that the formally chaotic solutions are physically unstable in the sense that their configurations disrupt rapidly, at most during the integration period of $7 \times 10^{4} \mathrm{yr}$ or $\sim 2 \times 10^{4} P_{c}$. Another conclusion is that one should not skip the stability test in the fitting procedure, as the procedure will not "see" the rapidly changing regions of the permitted (stable) initial conditions. To illustrate this issue, we computed the dynamical maps for a marginally worse solution than the best one [with $\left(\chi_{\nu}^{2}\right)^{1 / 2}=1.627$ and an rms of $\sim 10.32 \mathrm{~m} \mathrm{~s}^{-1}$; its orbital parameters are given in the caption to Fig. 2]. In this case the MMRs $4: 1,5: 1$, and $6: 1$ overlap, and the resulting stable zones are much wider than for the formal best fit. We note that in this solution, the value of $a_{c}$ is larger by about $0.1 \mathrm{AU}$ from the value of $a_{c}$ of the best-fit solution and can be found in a small clump of points in Figure 1 to the right of the main minimum. Yet the close coincidence between the $\log \mathrm{SN}$ and $\max e_{c}$ maps is still accurately preserved. This constitutes an excellent argument for the validity of the GAMP-like approach. Without it, stable solutions can basically be found only by chance.

In another search, we assumed that the system was not coplanar. We extended the model to 14 free parameters, including the orbital inclinations and one nodal longitude. As one would expect, the inclinations were barely constrained by the RV data; nevertheless, we found an interesting behavior of the HD 202206 system. There exist many solutions whose initial orbits have similar inclinations, but the relative inclination is not small: $i_{\text {rel }} \sim 94^{\circ}$. We selected one of these solutions for a closer analysis. Its parameters are given in the caption to Figure 3. The synthetic RV curves for both solutions (the coplanar and the mutually inclined configurations) can be barely distinguished one from another (see Fig. 4). The best-fit solution with the inclined orbits is also found in the zone of the 5:1 MMR (see Fig. 3). Again, the formal stability is closely related to the geometrical evolution of the orbits. 

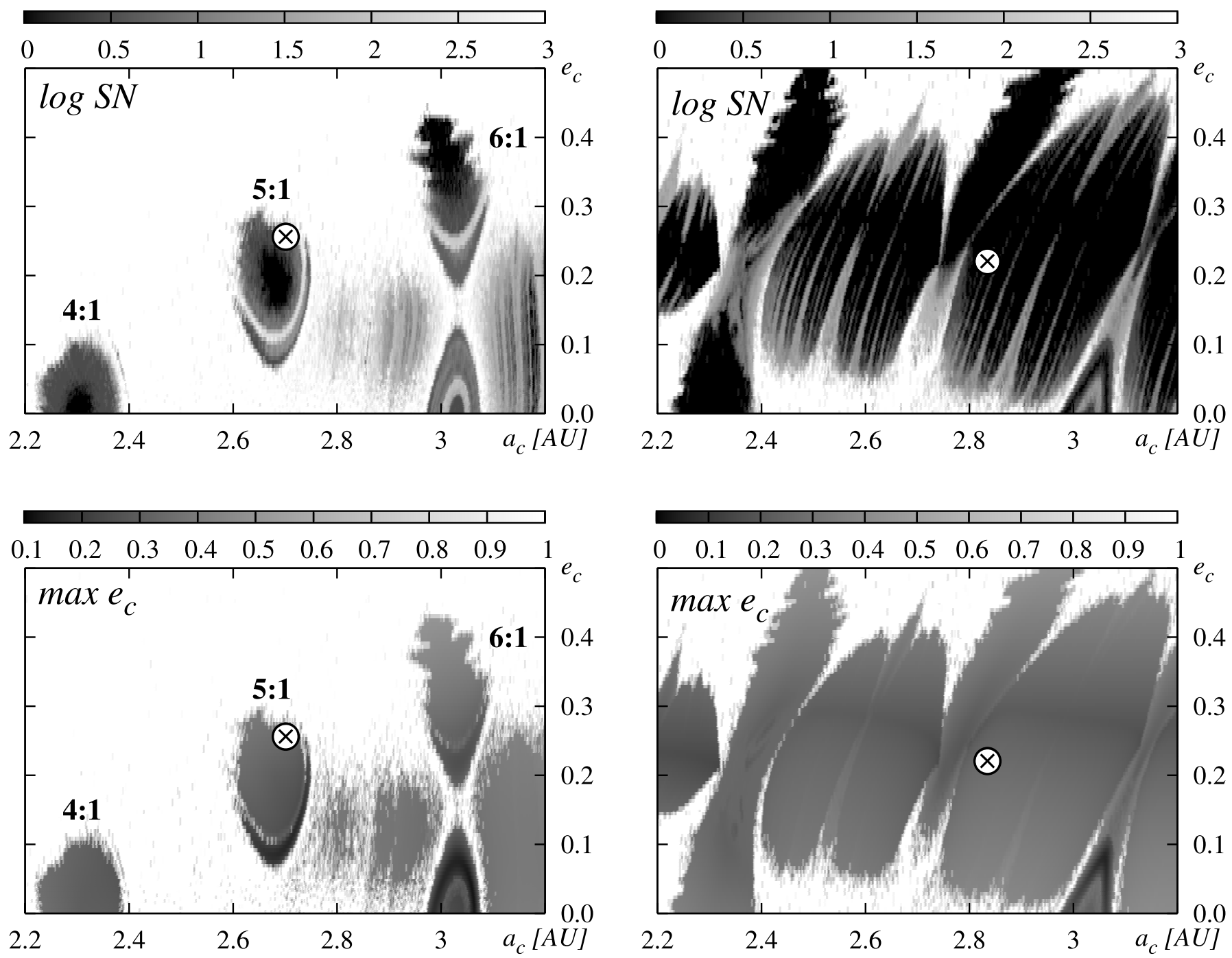

FIG. 2.-Left: Dynamical maps in the $\left(a_{c}, e_{c}\right)$-plane in terms of the spectral number, $\log \mathrm{SN}$, and max $e_{c}$ for a putative $5: 1$ MMR in a coplanar HD 202206 system (see Table 1). Shadings used in the log SN map classify the orbits: black indicates quasi-periodic, regular configurations, while white indicates strongly chaotic systems. A crossed circle marks the best-fit configuration. Right: Same as at left, but for a slightly worse initial condition with $\left(\chi_{\nu}^{2}\right)^{1 / 2}=1.62$ and an rms of $\sim 10.32 \mathrm{~m} \mathrm{~s} \mathrm{~s}^{-1}$. The osculating elements at the epoch of the first observation $\left(m\left[M_{\mathrm{J}}\right], a[\mathrm{AU}], e, \omega[\mathrm{deg}], \mathcal{M}[\mathrm{deg}]\right)$ are as follows: $(17.589,0.831,0.435,161.118,353.944)$ for planet $b$ and $(2.247,2.835,0.220,159.848,1.247)$ for planet $\mathrm{c}$, with $V_{0}=-0.47 \mathrm{~m} \mathrm{~s}^{-1}$. The resolution of the maps is $600 \times 120$ data points. Integrations are for $2 \times 10^{4}$ periods of the outer planet $\left(\sim 7 \times 10^{4} \mathrm{yr}\right)$. The islands of the relevant MMRs are labeled. [See the electronic edition of the Journal for a color version of this figure.]
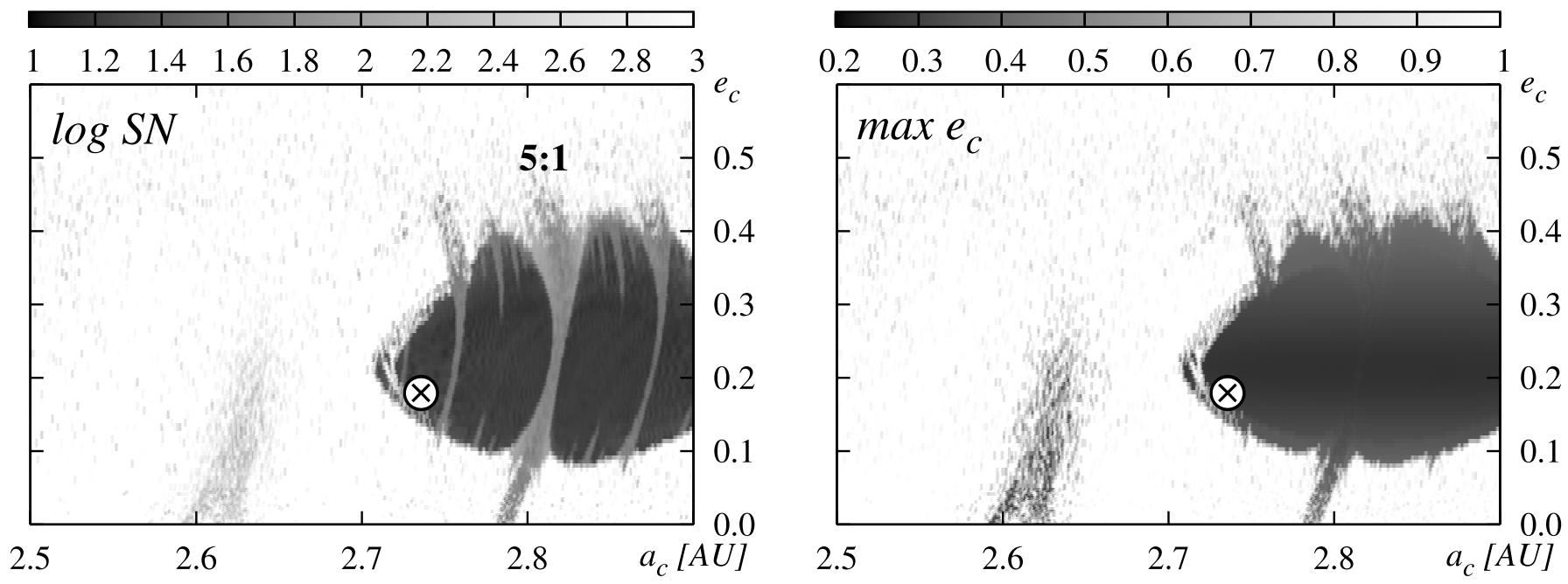

FIG. 3.-Dynamical maps in the $\left(a_{c}, e_{c}\right)$-plane in terms of the spectral number, $\log \mathrm{SN}$, and max $e_{c}$ for the best fit with mutually inclined orbits in the HD 202206 system. Shadings used in the log SN map classify the orbits: black indicates quasi-periodic, regular configurations, while white indicates strongly chaotic systems. A crossed circle denotes the best-fit configuration. The initial condition yields $\left(\chi_{\nu}^{2}\right)^{1 / 2}=1.59$, with an rms of $\sim 9.97 \mathrm{~m} \mathrm{~s}^{-1}$ (the number of fit parameters is 14). The osculating elements at the epoch of the first observation $\left(m\left[M_{\mathrm{J}}\right], a[\mathrm{AU}], e, i[\mathrm{deg}], \Omega[\mathrm{deg}], \omega[\mathrm{deg}], \mathcal{M}[\mathrm{deg}]\right)$ are as follows: $(17.723,0.831,0.435,83.625,265.307,161.040$, $353.921)$ for planet $\mathrm{b}$ and $(2.348,2.736,0.178,82.372,0.0,127.813,40.962)$ for planet $\mathrm{c}$, with $V_{0}=-1.78 \mathrm{~m} \mathrm{~s}^{-1}$. The resolution of the maps is $600 \times 120$ data points. Integrations are for $2 \times 10^{4}$ periods of the outer planet $\left(\sim 7 \times 10^{4} \mathrm{yr}\right)$. [See the electronic edition of the Journal for a color version of this figure.] 


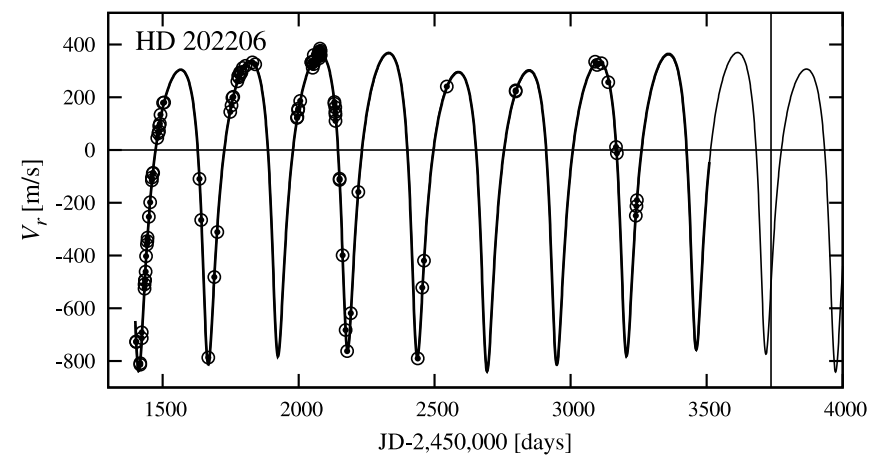

FIG. 4.-Synthetic RV curves for the HD 202,206 system. The thin line indicates a stable ( $N$-body) solution corresponding to a 5:1 MMR in the coplanar system, and the thick line indicates a 5:1 MMR in the configuration with mutually inclined orbits. Circles indicate the RV measurements published graphically in Correia et al. (2005).

Remarkably, the orbital evolution in these two cases is qualitatively different. This is illustrated in Figure 5. In the coplanar fit, as one would expect, the orbital eccentricity of the more massive planet stays close to the initial value, while the eccentricity of the outer planet varies with a large amplitude according to the conservation of the total angular momentum. In the mutually inclined configuration, the orbital evolution is quite unexpected. The eccentricity of the inner and larger planet varies with a much larger amplitude than the eccentricity of the smaller companion. Simultaneously, the inclination of the companion planet $\mathrm{c}$ spans almost the whole possible range. This example demonstrates a potential problem with a proper interpretation of the RV data. Since we do not know the true initial orbital inclinations, we cannot be sure about the choice of the best-fit configuration and hence the orbital evolution of the whole system. In the case of HD 202206, this issue is of special importance because the system may be considered a hierarchical one: the inner pair is the binary of the Sun-like star and a brown dwarf, and the outer planet is a Jovian compan-
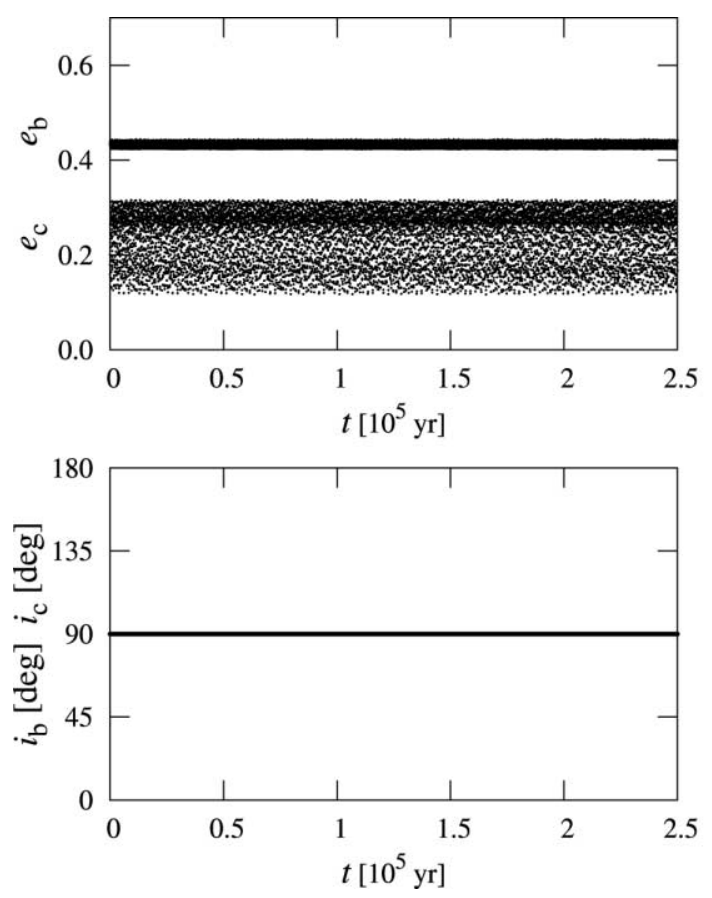

ion. In this sense, the HD 202206 system reminds us of a triple stellar system rather than a "usual" planetary system. In that case, the assumption of a coplanar configuration may be no longer valid. In addition, some recent works indicate that extrasolar planetary systems with mutually inclined orbits may be quite frequent (Thommes \& Lissauer 2003; Adams \& Laughlin 2003).

\section{A TREND IN THE RV DATA (14 HERCULIS)}

In many observed extrasolar planetary systems, linear trends in the RV data are present, indicating the existence of more distant companions. The GAMP may be very useful to constrain orbital parameters when the RV observations cover partially the longest orbital period. A good example is the $\mu$ Arae system (Jones et al. 2002; McCarthy et al. 2004). We did an extensive analysis of the available RV measurements of $\mu$ Ara in two earlier papers (Goździewski et al. 2003, 2005). The results of the first work, which were based on the RV data covering only a fragment of the orbital period of the outer planet, coincide remarkably with the outcome of the second analysis. In the later work, the observations cover about $70 \%$ of the orbital period of the putative outermost planet. We found that the stability constraints help to remove the artifacts, such as the extremely large eccentricity of the outer planet, and bound the space of permissible orbital parameters. Otherwise, the data permit a continuum of equally good orbital solutions.

The 14 Her system was announced by M. Mayor (1998, unpublished). The presence of a Jovian planet in this system was next confirmed by Butler et al. (2003) and Naef et al. (2004). In the later work, the discovery team found that the RV data have a linear slope of $\sim 3.6 \mathrm{~m} \mathrm{~s}^{-1} \mathrm{yr}^{-1}$. The single-planet Keplerian solution yields an abnormally large rms of about $14 \mathrm{~m} \mathrm{~s}^{-1}$. Even when the drift is accounted for, the rms of the single planet plus drift model leads to an rms of $\sim 11.3 \mathrm{~m} \mathrm{~s}^{-1}$, which is much larger than the mean observational uncertainties, $\sigma_{m} \sim 7.2 \mathrm{~m} \mathrm{~s}^{-1}$. Because the trend is similar to the one observed in the $\mu$ Ara data, we try to find a better solution with GAMP.
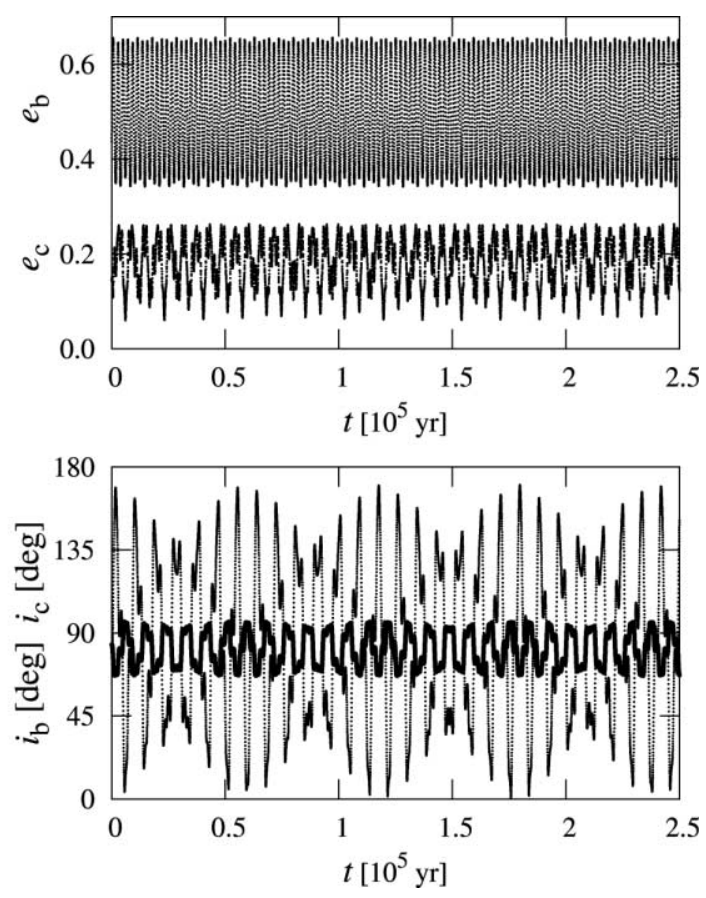

FIG. 5.- Orbital evolution of the HD 202,206 configurations corresponding to the best-fit coplanar solution (left; the elements are given in Table 1) and for the system with mutually inclined orbits (right; see the caption to Fig. 3 for the osculating elements of this configuration). 

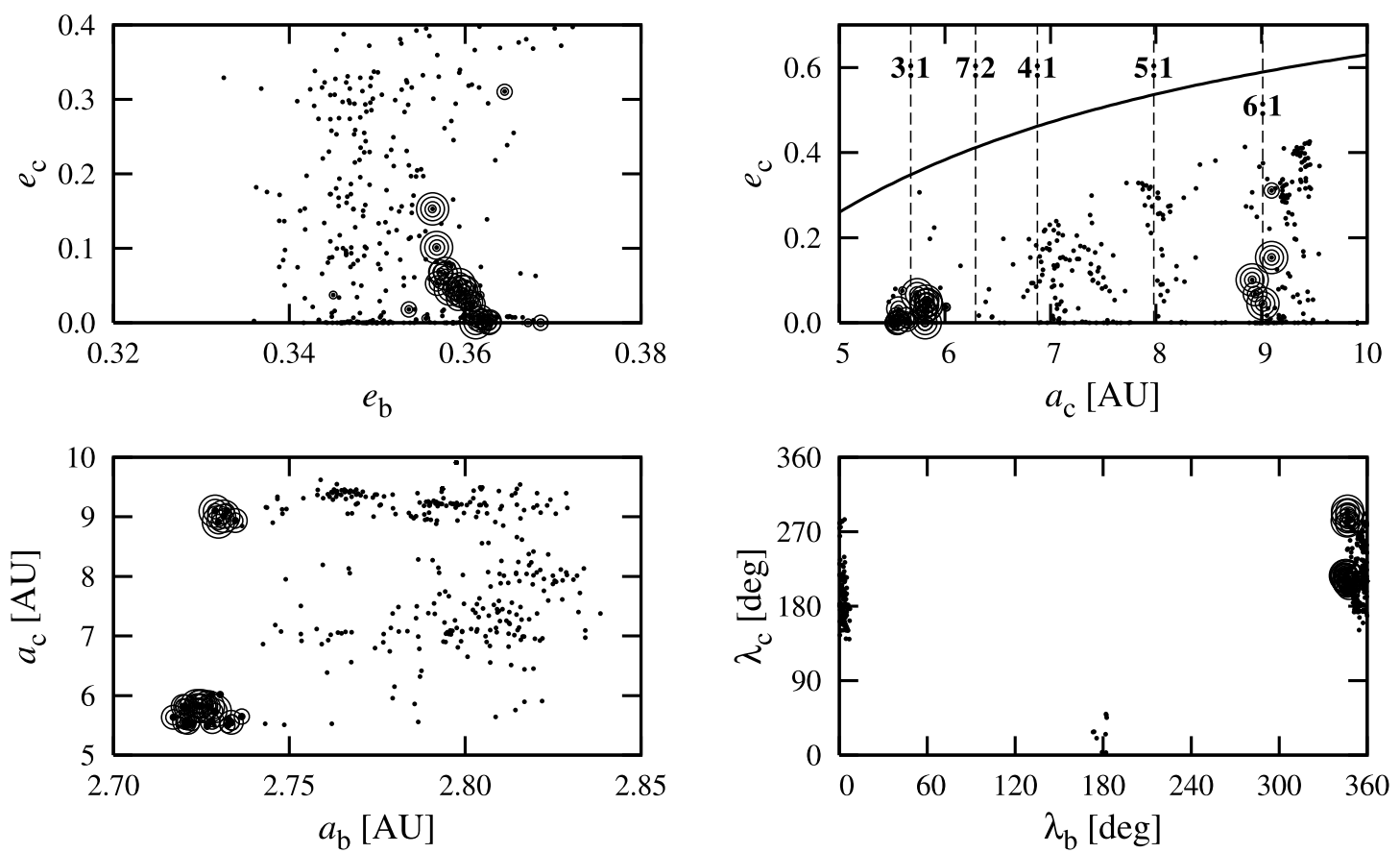

FIG. 6. - Best fits obtained with GAMP for the RV data published in Butler et al. (2003) and Naef et al. (2004) for 14 Her. The system is assumed to be coplanar. Parameters of the fit are projected onto the planes of osculating orbital elements at the epoch of the first observation, JD 2,449,464.5956. The smallest filled circles indicate stable solutions with $\left(\chi_{\nu}^{2}\right)^{1 / 2}<1.4$ and an rms of $\sim 11 \mathrm{~m} \mathrm{~s}^{-1}$. Larger open circles indicate $\left(\chi_{\nu}^{2}\right)^{1 / 2}=1.146,\left(\chi_{\nu}^{2}\right)^{1 / 2}<1.129$, and $\left(\chi_{\nu}^{2}\right)^{1 / 2}<1.117$, corresponding to $3 \sigma, 2 \sigma$, and $1 \sigma$ confidence intervals of the best-fit solution, respectively. The largest circles indicate the solutions with $\left(\chi_{\nu}^{2}\right)^{1 / 2}<1.111$, which is marginally larger than the value of $\left(\chi_{\nu}^{2}\right)^{1 / 2}=1.109$ of the two best fits given in Table 1. A curve in the $\left(a_{c}, e_{c}\right)$-plane indicates the planetary collision line. It is determined from the relation $a_{b}\left(1+e_{b}\right)=a_{c}\left(1-e_{c}\right)$, with $a_{b}$ and $e_{b}$ fixed at their best-fit values. The nominal positions of the most relevant MMR inferred from the Keplerian law are also marked by dashed lines and labeled.

Since 14 Her is a quiet star with $\log R_{\mathrm{HK}}^{\prime}=-5.07$ (Naef et al. 2004), it is thus reasonable to adopt a rather safe estimate of the stellar jitter, $\sigma_{j}=4 \mathrm{~m} \mathrm{~s}^{-1}$ (Wright 2005). Still, the rms exceeds the joint uncertainty $\sigma=\left(\sigma_{m}^{2}+\sigma_{j}^{2}\right)^{1 / 2} \sim 8 \mathrm{~m} \mathrm{~s}^{-1}$ by a few m s$~^{-1}$. Luckily, the RV data of this star are published and publicly available (Naef et al. 2004). There are 119 known observations. We combined these with 35 much more accurate measurements (with mean $\sigma_{m} \sim 3.1 \mathrm{~m} \mathrm{~s}^{-1}$ ) by the Carnegie Planet Search Program team (Butler et al. 2003) covering the middle part of the joint observational window. The full set consists of 154 measurements spanning about 3400 days and corresponding to about $2 P_{b}$. For the mass of the parent star, we adopted the value of $0.9 M_{\odot}$ (Naef et al. 2004).

We assume that the drift and the large residual signal are due to a long-period companion in the system. Using GAMP, we searched for a body in an orbit of $a_{c} \in(4,10) \mathrm{AU}$, trying to verify whether the available data could already be useful to constrain the orbital parameters of the putative distant planet. The results of thousands of independent GAMP runs are illustrated in Figure 6. Only the parameters of the stable best fits are projected onto selected planes of the osculating elements at the epoch of the first observation, JD 2,449,464.5956. The better the quality of the fits, as measured by their $\left(\chi_{\nu}^{2}\right)^{1 / 2}$, the larger the symbols in Figure 6. The largest circles indicate the best-fit solutions (given in Table 1) that have $\left(\chi_{\nu}^{2}\right)^{1 / 2} \sim 1.11$ with an $\mathrm{rms}$ of $\sim 8.5 \mathrm{~m} \mathrm{~s}^{-1}$. The smallest filled circles indicate the fits with $\left(\chi_{\nu}^{2}\right)^{1 / 2} \sim 1.4$, corresponding to the limit of an rms of $\sim 11 \mathrm{~m} \mathrm{~s}^{-1}$. Curiously, two well-defined local minima with almost the same value of $\left(\chi_{\nu}^{2}\right)^{1 / 2}$ are present. The synthetic RV curves for the best fits are illustrated in Figure 7 (all the available measurements are also marked with error bars). As we might have expected, the curves could not be distinguished from each other in the time range covered by the data. However, a clear choice between the curves could have been made already by the time of writing this paper (note that a vertical line at about JD 2,453,736.46 marks the end of the year 2005).

The two best fits correspond to qualitatively different configurations with $a_{c} \sim 5.8 \mathrm{AU}$ and $a_{c} \sim 9 \mathrm{AU}$. They are found in the proximity of the $3: 1 \mathrm{MMR}(14 \mathrm{Her}$ a) and the $6: 1 \mathrm{MMR}$ (14 Her b), respectively (see Fig. 6, top right; see also Table 1). Simultaneously, the parameters of the inner planet, as well as the relative phases of the companions, are already constrained very well. This makes it possible to perform a representative test of the system stability. We calculated two maps centered at the values of $a_{c}$ in the best fits, in the $\left(a_{c}, e_{c}\right)$-plane, keeping other orbital elements fixed at their best-fit values. The results are illustrated in Figure 8: the panels in the left column show the best fit for 14 Her a (marked by a crossed circle in each panel), and the panels in the right column show the best fit for 14 Her b. Clearly, the dynamical map of $\log \mathrm{SN}$ strongly coincides with the physical stability (in terms of max $e_{c}$ ) in the region spanned by the low-order resonances $3: 1,7: 2$, and $4: 1$. The best fit for $14 \mathrm{Her}$ a is found close to the border of the $3: 1 \mathrm{MMR}$. This is illustrated in Figure 9 for an initial condition that is close to the best one. It shows, in subsequent panels, time evolution of the eccentricities, the angle of the secular resonance $\theta$, and one of the critical arguments of the 3:1 MMR $\left(\theta_{31}=3 \lambda_{c}-\lambda_{b}-\varpi_{b}-\varpi_{c}\right)$. A perfect convergence of $\langle Y\rangle(t)$ confirms that the configuration is close to a quasiperiodic, ordered motion. The zone of stable motions extends up to the proximity of the collision zone, which is marked by a solid line in the maps. We note also the very sharp borders of the stability regions. Outside these zones, the configurations disrupt catastrophically, which is indicated by the values of $e_{c}$ increasing to 1 during at most $10^{5} \mathrm{yr}$ (the integration time). Obviously, in such a 
TABLE 1

Osculating, Astrocentric Elements of the Best Fits

\begin{tabular}{|c|c|c|c|c|c|c|c|c|c|c|c|}
\hline \multirow[b]{2}{*}{ PARAMETER } & \multicolumn{2}{|c|}{ HD 202206} & \multicolumn{2}{|c|}{14 Her a } & \multicolumn{2}{|c|}{14 Her b } & \multicolumn{3}{|c|}{ HD 37124} & \multicolumn{2}{|c|}{ HD 108874} \\
\hline & $\mathrm{b}$ & $\mathrm{c}$ & $\mathrm{b}$ & $\mathrm{c}$ & $\mathrm{b}$ & $\mathrm{c}$ & $\mathrm{b}$ & $\mathrm{c}$ & $\mathrm{d}$ & $\mathrm{b}$ & $\mathrm{c}$ \\
\hline$m \sin i\left(M_{\mathrm{J}}\right) \ldots \ldots \ldots \ldots \ldots \ldots$ & 17.624 & 2.421 & 4.485 & 2.086 & 4.533 & 6.289 & 0.624 & 0.574 & 0.695 & 1.358 & 1.008 \\
\hline$a(\mathrm{AU})$ & 0.831 & 2.701 & 2.727 & 5.810 & 2.730 & 8.911 & 0.519 & 1.610 & 3.142 & 1.051 & 2.658 \\
\hline e & 0.433 & 0.255 & 0.361 & 0.004 & 0.357 & 0.101 & 0.079 & 0.150 & 0.297 & 0.068 & 0.252 \\
\hline$\omega(\operatorname{deg})$ & 161.41 & 92.73 & 22.98 & 197.17 & 22.88 & 62.97 & 138.09 & 268.88 & 269.38 & 255.76 & 16.65 \\
\hline $\mathcal{M}(\mathrm{deg}) \ldots \ldots \ldots \ldots \ldots \ldots \ldots$ & 353.44 & 65.76 & 322.94 & 17.68 & 323.78 & 227.45 & 259.36 & 109.49 & 123.92 & 13.26 & 32.08 \\
\hline$\left(\chi_{\nu}^{2}\right)^{1 / 2} \ldots \ldots \ldots \ldots \ldots \ldots \ldots \ldots \ldots \ldots \ldots \ldots$ & \multicolumn{2}{|c|}{1.53} & \multicolumn{2}{|c|}{1.11} & \multicolumn{2}{|c|}{1.11} & \multicolumn{3}{|c|}{0.94} & \multicolumn{2}{|c|}{0.71} \\
\hline $\mathrm{rms}\left(\mathrm{m} \mathrm{s}^{-1}\right) \ldots \ldots \ldots \ldots \ldots \ldots$ & \multicolumn{2}{|c|}{9.98} & \multicolumn{2}{|c|}{8.53} & \multicolumn{2}{|c|}{8.51} & \multicolumn{3}{|c|}{3.38} & \multicolumn{2}{|c|}{3.30} \\
\hline$V_{0}\left(\mathrm{~m} \mathrm{~s}^{-1}\right) \ldots \ldots \ldots \ldots \ldots \ldots$ & \multicolumn{2}{|c|}{-1.36} & \multicolumn{2}{|c|}{-14.81} & \multicolumn{2}{|c|}{-55.65} & \multicolumn{3}{|c|}{7.72} & \multicolumn{2}{|c|}{17.28} \\
\hline$V_{1}\left(\mathrm{~m} \mathrm{~s}^{-1}\right) \ldots \ldots \ldots \ldots \ldots \ldots$ & \multicolumn{2}{|c|}{$\ldots$} & \multicolumn{2}{|c|}{-49.76} & \multicolumn{2}{|c|}{-90.48} & \multicolumn{3}{|c|}{$\ldots$} & \multicolumn{2}{|c|}{$\ldots$} \\
\hline p & \multicolumn{2}{|c|}{11} & \multicolumn{2}{|c|}{12} & \multicolumn{2}{|c|}{12} & \multicolumn{3}{|c|}{16} & \multicolumn{2}{|c|}{11} \\
\hline$M_{\star}\left(M_{\odot}\right) \ldots \ldots \ldots \ldots \ldots \ldots \ldots$ & \multicolumn{2}{|c|}{1.15} & \multicolumn{2}{|c|}{0.90} & \multicolumn{2}{|c|}{0.90} & \multicolumn{3}{|c|}{0.78} & \multicolumn{2}{|c|}{0.99} \\
\hline 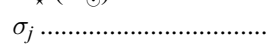 & & & & & & & & 3.2 & & & \\
\hline
\end{tabular}

Noтеs.-Data are given at the epoch of the relevant first observation. All systems are assumed to be coplanar and edge-on. Formal estimates of the uncertainties

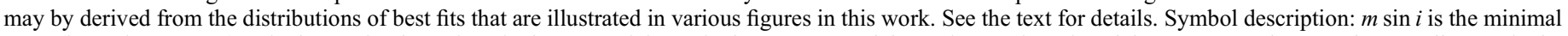
mass (in Jupiter masses), $a$ is the semimajor axis, $e$ is the eccentricity, $\omega$ is the argument of the pericenter in units of degrees, $V_{0}$ and $V_{1}$ are the RV offsets, $p$ is the number of fit parameters in the $N$-body model, $M_{\star}$ is the mass of the parent star, and $\sigma_{j}$ is the jitter estimate added in quadrature to the measurement errors.

case, if we used the pure $N$-body model of the RV, we would not be properly handling the sophisticated, discontinuous structure of the phase space.

This last conclusion is also valid for the solution for $14 \mathrm{Her} b$ with the more distant planet (see Fig. 8, right). We note an excellent coincidence of the distribution of the best fits (Fig. 6) with the stable areas unveiled by the SM in Figure 8 . The space in the range $[7,9] \mathrm{AU}$ is spanned by a few low-order MMRs $(4: 1,5: 1$, and $6: 1)$ of varying width, which are already overlapping for values of $e_{c}$ that are $\sim 0.2$ less than the values determined by the equation of the collision line. The border of the stability areas is sharp. Formally chaotic configurations would be quickly disintegrated by collisions or ejection of a companion from the system (see the relevant max $e_{c}$ map in Fig. 8). Curiously, the best fit is located between the $11: 2$ and $6: 1$ MMRs, which reminds us of the HD 12661 planetary system (Fischer et al. 2003; Goździewski \& Maciejewski 2003).

According to the above investigations, it remains very likely that the 14 Her system hosts two Jovian planets involved in a low-order MMR. Let us remark that the presence of the two wellseparated local minima of $\left(\chi_{\nu}^{2}\right)^{1 / 2}$ is not so clear when both the $\mathrm{RV}$ data sets are analyzed separately. At present, even if more

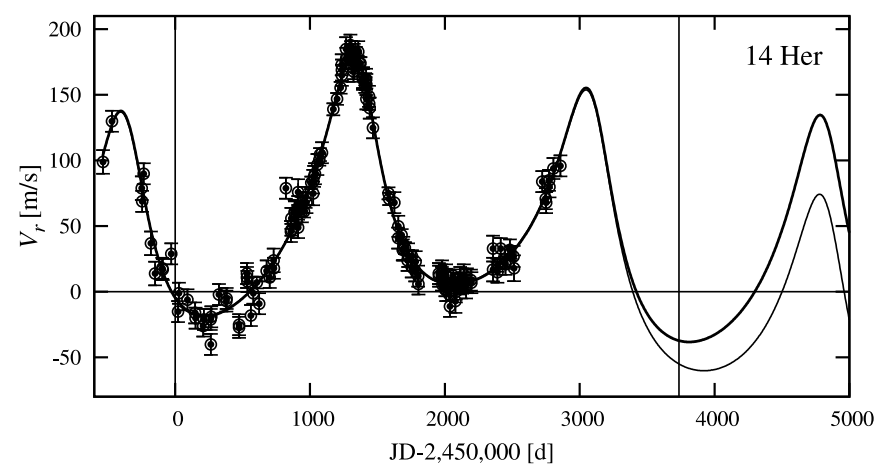

FIG. 7.-Synthetic RV curves for the two best-fit solutions (see Table 1) to the RV data of 14 Her. The thick line indicates 14 Her a (a proximity to the $3: 1 \mathrm{MMR}$ ), and the thin line indicates 14 Her b (around the $6: 1 \mathrm{MMR}$ ). Data points are plotted with error bars indicating the joint RV error (stemming from the errors of measurements and the stellar jitter of $4 \mathrm{~m} \mathrm{~s}^{-1}$ added in quadrature). The vertical line at JD 2,453,736 indicates the calendar date 2005 December 31 . data for the 14 Her system were available, it would be desirable to search for the best fits with a GAMP-like algorithm. Contrary to the impression caused by the presence of the apparent longterm drift in the data, a few recent measurements may already be helpful to resolve a plausible orbital configuration of the $14 \mathrm{Her}$ system. Yet the measurements gathered by the two observing teams are in excellent accord, and the data sets complement each other.

\section{A MULTIPLANET CONFIGURATION (HD 37124)}

Recently, Vogt et al. (2005) announced a discovery of several multiplanet systems. In particular, the previously known twoplanet system about HD 37124 is thought to harbor one more planet. A hypothesis of the third planet removes a previously present degeneracy of the two-planet solution to the RV that allowed a large eccentricity of the outer planet and collisional destabilization of the system (Goździewski 2003). The discovery team found that the double-Keplerian model of the RV reveals two best-fit solutions of similar quality. In the better one, the planets revolve in almost circular orbits with periods of about 155,843 , and 2300 days, respectively. Curiously, in this solution the Keplerian periods $P_{d} \sim 3 P_{c}$ may indicate a proximity of the system to a low-order resonance. A possibility of such low-order commensurability warns that the application of the Keplerian models of the RV is problematic. Indeed, an inspection of the three-planet Keplerian best-fit solutions reveals that they correspond to strongly chaotic motions and that the system would easily disintegrate through mutual interactions. The triple-Keplerian initial condition found by Vogt et al. (2005) has a fixed value of $e_{d}=0.2$, which was chosen to fulfill the requirement of dynamical stability.

Since HD 37124 is a quiet star with low activity index, $\log R_{\mathrm{HK}}^{\prime}=$ -4.90 (Vogt et al. 2005), the stellar jitter is likely small. We follow the discovery team by adopting $\sigma_{j}=3.2 \mathrm{~m} \mathrm{~s}^{-1}$. The instrumental errors have been rescaled by adding the value of jitter in quadrature to the measurement uncertainties. As a reference to further analysis, we reanalyzed the measurements using our hybrid GA-simplex code (Goździewski \& Migaszewski 2006), driven by the Keplerian model of the RV. The hybrid algorithm repeatedly converges to the best three-planet solution, which yields $\left(\chi_{\nu}^{2}\right)^{1 / 2}=0.873$ and an rms of $3.22 \mathrm{~m} \mathrm{~s}^{-1}$. The model 

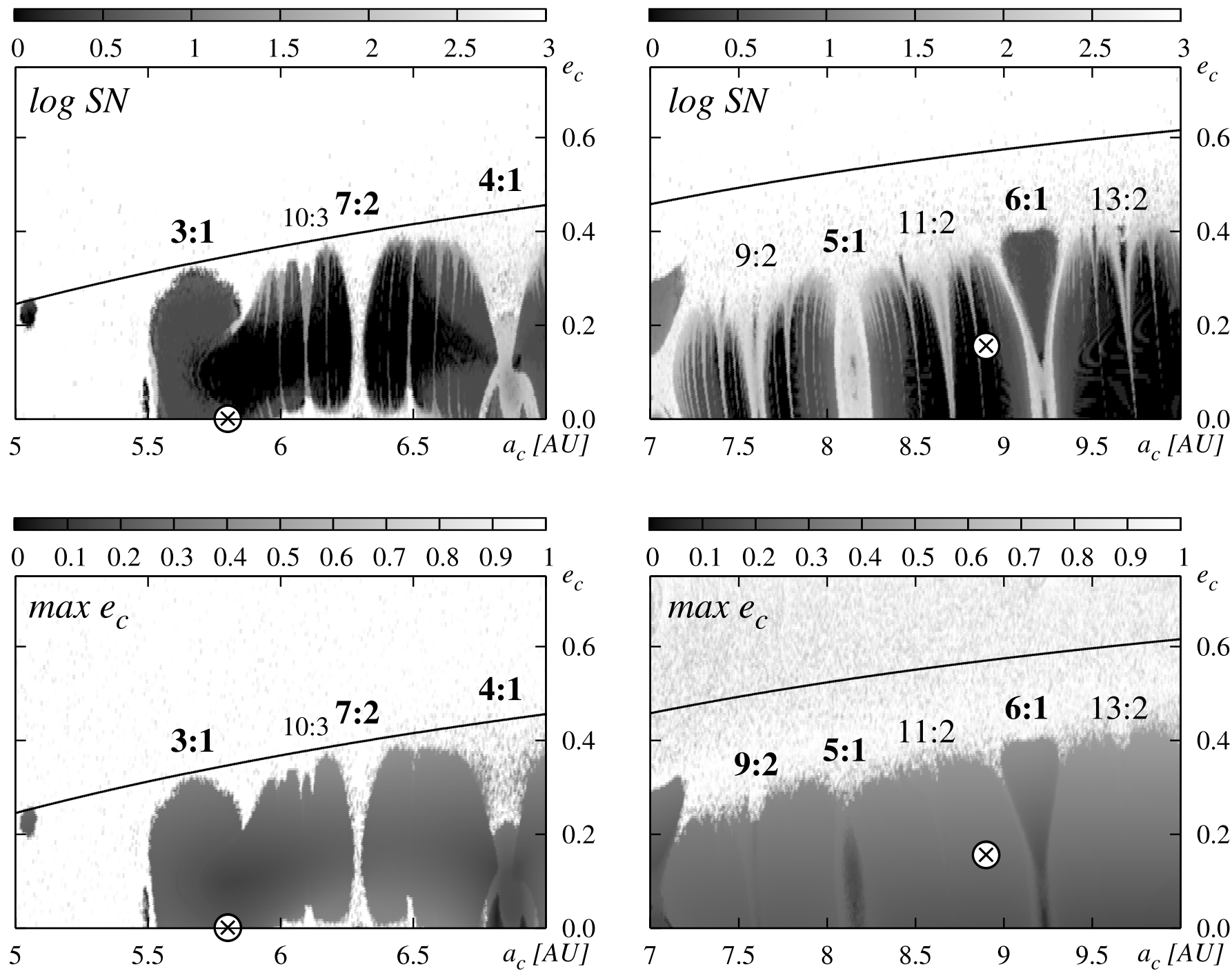

FIG. 8.-Stability maps in the $\left(a_{c}, e_{c}\right)$-plane in terms of the spectral number, $\log \mathrm{SN}$, and max $e_{c}$ for the best-fit solution to the RV data of the $14 \mathrm{Her}$ system. Shadings used in the $\log \mathrm{SN}$ map classify the orbits: black indicates quasi-periodic, regular configurations, while white indicates strongly chaotic systems. The crossed circles mark the best-fit configurations. The left panels show the fit to $14 \mathrm{Her}$ a, and the right panels show the fit to $14 \mathrm{Her} b$ (see Table 1). The relevant MMRs are labeled. A collision line calculated according to the formulae given in the caption to Fig. 6 is also marked for fixed best-fit elements of the inner planet. The resolution of the maps is $600 \times 120$ data points. Integrations are for $2 \times 10^{4}$ periods of the outer planet $\left(\sim 7 \times 10^{4} \mathrm{yr}\right)$. [See the electronic edition of the Journal for a color version of this figure.]

parameters $\left(K, P, e, \omega, T_{p}-T_{0}\right)$; that is, the semiamplitude, the orbital period, the eccentricity, the argument of periastron, and the time of periastron passage, for this fit are $\left(27.665 \mathrm{~m} \mathrm{~s}^{-1}\right.$, 154.495 days, $0.076,140.451,11853.604$ days $),\left(14.437 \mathrm{~m} \mathrm{~s}^{-1}\right.$, 854.330 days, $0.052,10^{\circ} .476,12072.944$ days $)$, and $\left(15.012 \mathrm{~m} \mathrm{~s}^{-1}\right.$, 2194.094 days, $0.456,293^{\circ} .496,12003.128$ days) for the inner, middle, and outer planets, respectively; $T_{0}=$ JD 2, 440,000, and the velocity offset is $V_{0}=8.058 \mathrm{~m} \mathrm{~s}^{-1}$. This solution is slightly better than the triple-Keplerian fit quoted by Vogt et al. (2005), but our solution has a much larger eccentricity: $e_{d} \sim 0.46$. The fit parameters of multiplanet systems are best interpreted in terms of osculating Keplerian elements and minimal masses related to the Jacobi coordinates (Lee \& Peale 2003). Adopting the date of the first observation as the osculating epoch, we recalculated the inferred astrocentric osculating elements $\left(m_{p} \sin i, a, e, \omega, \mathcal{M}\right)$ as follows: $\left(0.618 M_{\mathrm{J}}, 0.522 \mathrm{AU}, 0.075,137^{\circ} .890,262^{\circ} .142\right),\left(0.570 M_{\mathrm{J}}\right.$, 1.622 AU, $\left.0.052,10^{\circ} .476,23^{\circ} .499\right)$, and ( $0.725 M_{\mathrm{J}}, 3.060 \mathrm{AU}, 0.454$, $294^{\circ} .147,99^{\circ} .389$ ) for the planets $\mathrm{b}$, c, and d, respectively.

Remarkably, the hybrid code finds the best-fit solution without being given any a priori or additional information. One has only to determine some reasonable limits for the model parameters. Specifically, for the HD 37124 data, we explored the orbital periods in the range $[5,2500]$ days and eccentricities in the range $[0,0.8]$. Yet the apparently very precise three-planet Keplerian fit describes a collisional configuration, so we are certainly not done yet.

Next we reanalyzed the RV data with GAMP by conducting two searches. Keeping in mind the results of the Keplerian-based analysis done by the discovery team and the results of our search, we assumed in the first trial that the companions' orbits have moderate eccentricities in the range of $[0,0.5]$ and semimajor axes in safe enough bounds of $a_{b} \in[0.4,0.8] \mathrm{AU}, a_{c} \in[1.0,1.8]$ $\mathrm{AU}$, and $a_{d} \in[2.0,4.0] \mathrm{AU}$. The MEGNO was computed for the whole system over $\sim 2 \times 10^{3}$ periods of the outermost planet. The results of that GAMP search are illustrated in Figure 10, which shows the projections of the best-fit parameters onto the planes of osculating elements at the epoch of the first observation. The quality of the gathered solutions is marked by the size of the symbols. The smallest filled circles indicate fits with $\left(\chi_{\nu}^{2}\right)^{1 / 2}<$ 1.14; that is, within the $3 \sigma$ confidence interval of the best-fit 

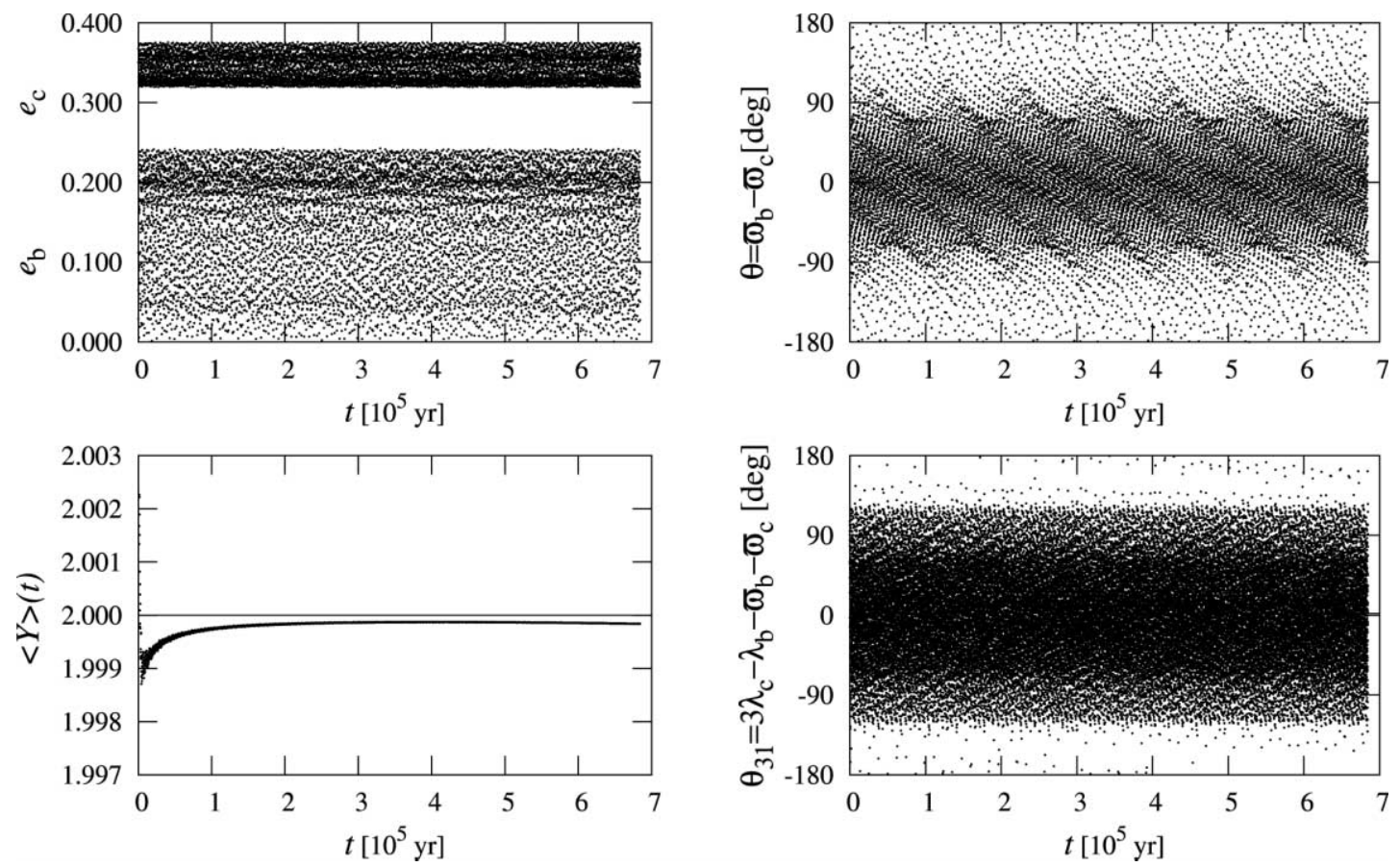

Fig. 9.- Orbital evolution of a configuration close to the best-fit solution of 14 Her a (see Table 1). The osculating elements for the epoch of the observations in the fits, given in terms of $\left(m\left[M_{\mathrm{J}}\right], a[\mathrm{AU}], e, \omega[\mathrm{deg}], \mathcal{M}[\mathrm{deg}]\right)$, are $(4.478,2.726,0.363,23.28,322.706)$ for planet $\mathrm{b}$ and $(1.945,5.628,0.0028,192.98,15.33)$ for planet $\mathrm{c}$, with $V_{0}=-13.38, V_{1}=-48.18 \mathrm{~m} \mathrm{~s}^{-1},\left(\chi_{\nu}^{2}\right)^{1 / 2}=1.111$, and an rms of $8.53 \mathrm{~m} \mathrm{~s}^{-1}$. From top to bottom and from left to right, we plot the eccentricities, the angle $\theta$ of the secular alignment of the apsides, the MEGNO $\langle Y\rangle$, indicating a quasi-regular configuration, and the critical argument of the 3:1 MMR.

solution given in Table 1. It appears that the single minimum of $\left(\chi_{\nu}^{2}\right)^{1 / 2}$ that we found is quite precisely determined. The elements of the innermost planets are already very well constrained. For instance, the semimajor axis of the companion planet $b$ changes within only $0.001 \mathrm{AU}$ at the $1 \sigma$ confidence interval of the best-fit solution. Obviously, the largest uncertainties are for the outermost companion planet $\mathrm{d}$, but even in this case, the errors do not seem to be large.

Yet the apparently well-constrained minimum of $\left(\chi_{\nu}^{2}\right)^{1 / 2}$ is localized in a region of the phase space that has a very complex dynamical structure. This is illustrated in Figure 11, which shows the dynamical maps in the $\left(a_{d}, e_{d}\right)$-plane. These maps are computed for a few initial conditions chosen from the set of the best fits illustrated in Figure 10. The relevant initial conditions are marked in the dynamical maps by large crossed circles. The top left panel of Figure 11 shows the best Newtonian solution obtained without the MEGNO penalty. Mathematically, that fit is the best one, as it has a $\left(\chi_{\nu}^{2}\right)^{1 / 2} \sim 0.86$, with an rms of $\sim 3.11 \mathrm{~m} \mathrm{~s}^{-1}$, a little better than the Keplerian best-fit solution quoted above. Nevertheless, this fit is also unacceptable because it lies very close to the collision zone of the two outermost orbits, which is marked in the figure by a smooth curve. In this area the motions are strongly chaotic and unstable. Far below the collision line, we identify the most relevant MMRs of these planets: $7: 3,5: 2$, $8: 3$, and $3: 1$, at the very edge of the map. In the top right panel of Figure 11, we chose a relatively good initial condition with $\left(\chi_{\nu}^{2}\right)^{1 / 2} \sim 1.25$ and an $\mathrm{rms}$ of $\sim 4.4 \mathrm{~m} \mathrm{~s}^{-1}$, which is located in the proximity of the $7: 3 \mathrm{MMR}$. Note a significant change of the shape of the $5: 2 \mathrm{MMR}$ as compared with the top left panel. Some fits at the $1 \sigma-2 \sigma$ confidence levels of $\left(\chi_{\nu}^{2}\right)^{1 / 2}$ may fall into the libration zone of this MMR, and they have quite large initial values of $e_{d} \sim 0.3$. An example is illustrated in Figure 12. The configuration is formally chaotic, but the critical angles $\theta=\varpi_{d}-\varpi_{c}$ and $\theta_{52}=5 \lambda_{d}-2 \lambda_{c}-3 \varpi_{d}$ librate about $180^{\circ}$; the eccentricities do not exhibit any secular changes over $3 \mathrm{Myr}$ of integration. Note that in this case MEGNO stays close to 2 for about 0.3 Myr, the stability criterion used in GAMP was not violated, and the weakly chaotic configuration has been left in the set of acceptable solutions.

The left panel in the middle row of Figure 11 shows the fit with $\left(\chi_{\nu}^{2}\right)^{1 / 2}=1.11$ and an rms of $\sim 4 \mathrm{~m} \mathrm{~s}^{-1}$ and a small initial eccentricity of the outermost planet. On the right, an even better solution with $\left(\chi_{\nu}^{2}\right)^{1 / 2}=0.96$ is related to a configuration between the $5: 2$ and $8: 3$ MMR. The bottom left panel of Figure 11 shows a close-up of the dynamical neighborhood of the stable best-fit solution (see Table 1). This fit lies close to the border of the $11: 4 \mathrm{MMR}$. In this zone, higher order resonances and the fine structure of the phase space are also visible. Finally, the bottom right panel shows the dynamical map corresponding to the initial condition, which yields $\left(\chi_{\nu}^{2}\right)^{1 / 2} \sim 1$ and an rms of $\sim 3.62 \mathrm{~m} \mathrm{~s}^{-1}$. It would correspond to a system locked in the 11:4 MMR of the outermost planets. In this panel, for a reference, we also marked the solutions with $\left(\chi_{\nu}^{2}\right)^{1 / 2}<1$, roughly corresponding to the $2 \sigma$ confidence interval of the best-fit solution in Table 1. Actually, many fits found in this zone and resolved with a relatively short integration time of MEGNO, $\sim 2000 P_{c}$, appear to be weakly chaotic.

Let us note that the best-fit initial condition given in Table 1 has been refined through a postfitting analysis with the MEGNO computed over 50,000 periods of the outermost planet. The MEGNO for this fit converges to 2 well over 12 Myr (Fig. 13). This indicates a strictly regular (quasi-periodic) orbital evolution. All eccentricities change with significant amplitudes of $\sim 0.1-0.3$. This is illustrated in Figure 13. A secular apsidal resonance (SAR) with a small semiamplitude of about $20^{\circ}$ is present in the motion of the two outermost planets. The synthetic RV curve of the best stable fit is shown in Figure 14.

In the relevant region of the $\left(a_{d}, e_{d}\right)$-plane, the positions of the MMRs, as well as their widths, vary in the range of $\sim 0.2 \mathrm{AU}$ with respect to $a_{d}$ when the initial conditions are changed. The 

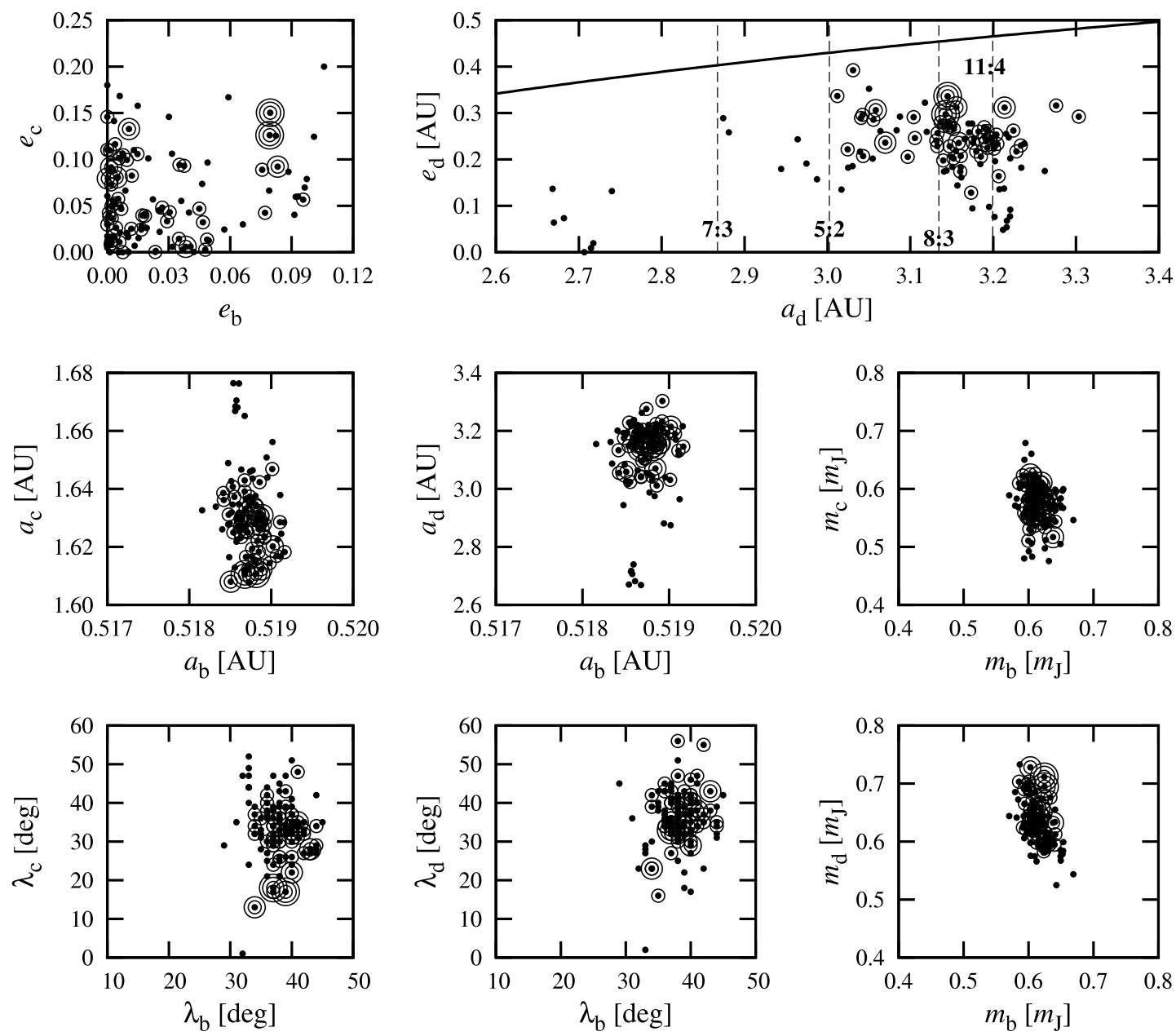

FIG. 10.-Best fits obtained with the GAMP for the RV data published in Vogt et al. (2005) of HD 37124. The system is assumed to be coplanar. Parameters of the fit are projected onto the planes of the osculating orbital elements at the epoch of the first observation, JD 2,450,420.047. The smallest filled dots indicate GAMP solutions with $\left(\chi_{\nu}^{2}\right)^{1 / 2}<1.1$ and an rms of $\sim 4.0 \mathrm{~m} \mathrm{~s}^{-1}$. Larger open circles indicate $\left(\chi_{\nu}^{2}\right)^{1 / 2}=1.02$ and $\left(\chi_{\nu}^{2}\right)^{1 / 2}=0.97(2 \sigma$ and $\sim 1 \sigma$ confidence intervals of the best-fit solution, respectively). The largest circles indicate the solutions with $\left(\chi_{\nu}^{2}\right)^{1 / 2}<0.94$, marginally larger than the value of $\left(\chi_{\nu}^{2}\right)^{1 / 2}=0.935$ of the best fit found in the whole search. In the top right panel, a curve in the $\left(a_{d}, e_{d}\right)$-plane indicates the planetary collision line for the outermost companions. It is determined from the relation $a_{c}\left(1+e_{c}\right)=a_{d}\left(1-e_{d}\right)$, with $a_{c}$ and $e_{c}$ fixed at their best-fit values. The nominal positions of the most relevant MMRs inferred from the Keplerian law are also marked by dashed lines and labeled.

border of the zone of global instability is highly irregular but very sharp and, as one would expect, it can be found in the max $e$ maps (not shown here). A conclusion provided by this experiment is that the structure of the phase space changes dramatically, even if we choose initial conditions that are statistically comparable and relatively close to each other. An inspection of the dynamical maps in Figure 11 reveals that it is hardly possible to avoid the unstable areas without explicitly accounting for the stability criterion in a self-consistent manner. One might think that the $\mathrm{N}$-body model does not lead to a significant improvement of $\left(\chi_{\nu}^{2}\right)^{1 / 2}$; we obtained very similar values, $\left(\chi_{\nu}^{2}\right)^{1 / 2} \sim 0.88-0.96$, to those of the best fits found with the triple-Keplerian model of the RV. Nevertheless, both the Keplerian and Newtonian best-fit solutions obtained without the stability check are in some sense degenerate because they yield precise but physically unacceptable, disrupting configurations.

The best fits found in the GAMP search and the result of the dynamical analysis reveal an intriguing state of the HD 37124 system. It resides in a dynamically very active region of the phase space. It remains possible that the two external planets are close to the $5: 2 \mathrm{MMR}$, similar to the Jupiter-Saturn case. We found some acceptable fits within the libration island of this resonance; however, in such a case the eccentricities of both of the outermost companions would be relatively large at $\sim 0.3$ (see Fig. 12). Some best-fit configurations are very close to the $8: 3$ or 11:4 MMRs. As we demonstrate by the computations illustrated in Figure 11, the parameters' errors bounds are relatively extended and the proximity of the system to any of these resonances cannot be excluded at present. In this sense, with the currently available observations it is not possible to choose one particular configuration that could represent the true state of the system.

In the second GAMP search we looked for the best fits, assuming that the semimajor axes were of about $a_{b} \in[0.05,0.3] \mathrm{AU}$, $a_{c} \in[0.3,0.6] \mathrm{AU}$, and $a_{d} \in[1.2,1.8] \mathrm{AU}$. In this way we tried to verify the Keplerian fits of the second plausible configuration found by the discovery team. Their analysis reveals the best-fit solution, which could be concurrent with the configuration with the long-period orbit of companion planet $d$ but which has a significantly worse $\left(\chi_{\nu}^{2}\right)^{1 / 2} \sim 1.14$ (Vogt et al. 2005). The GAMPresolved $N$-body solutions are also not so good, as for the previously analyzed configurations. The best fit found in the GAMP search has $\left(\chi_{\nu}^{2}\right)^{1 / 2} \sim 1.2$. In that case, the innermost planet would be a hot Neptune with a mass of about $0.1 M_{\mathrm{J}}$ and a semimajor axis of about $0.1 \mathrm{AU}$. Overall, this fit is even worse than the tripleKeplerian fit found by the discovery team, with $\left(\chi_{\nu}^{2}\right)^{1 / 2} \sim 1.14$. 

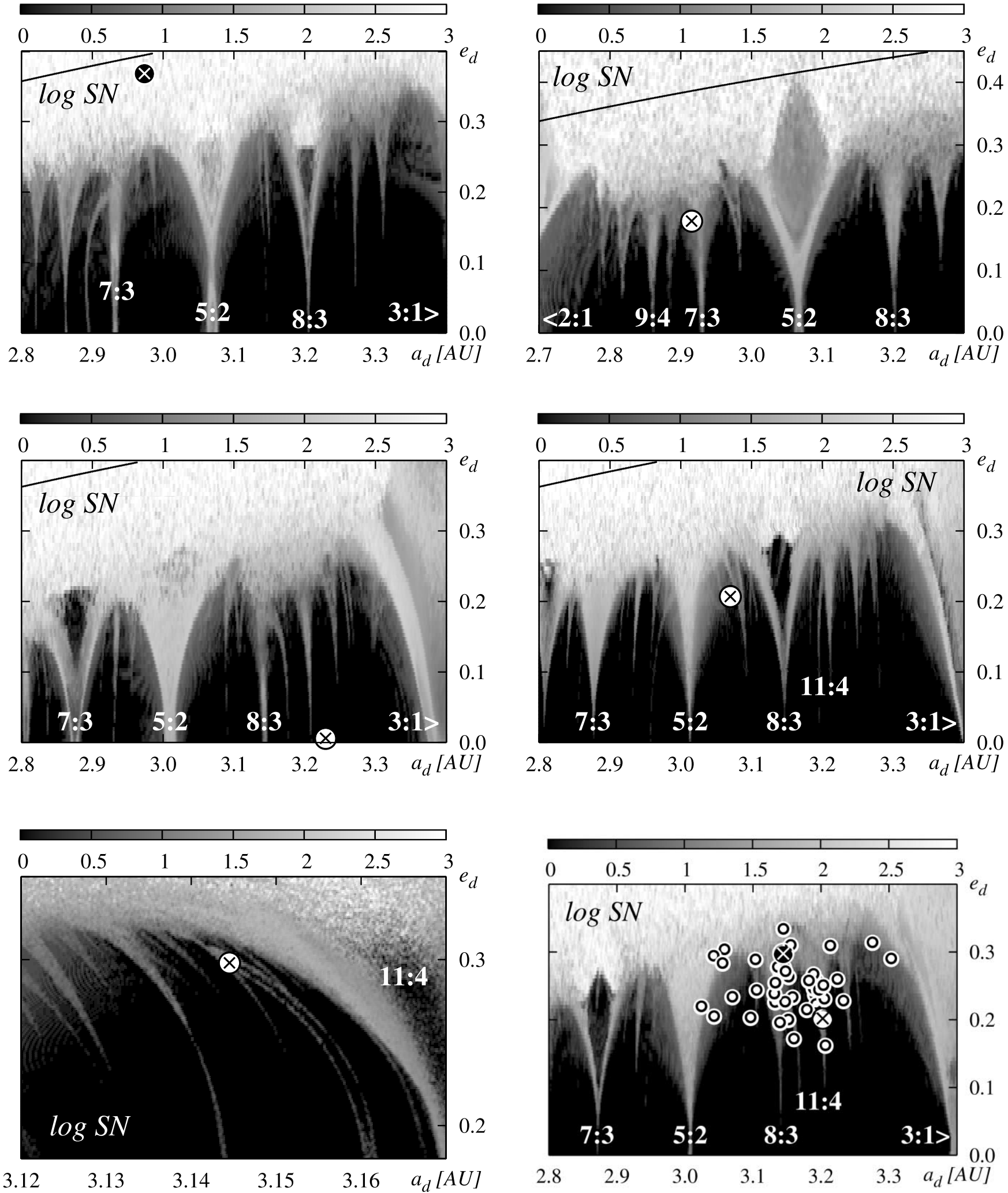

FIG. 11.-Dynamical maps in the $\left(a_{d}, e_{d}\right)$-plane for the best-fit solutions to the RV data of HD 37124 . The shading scheme is the same as in Fig. 8. The initial conditions,

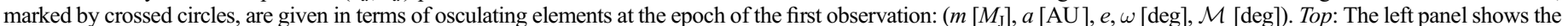

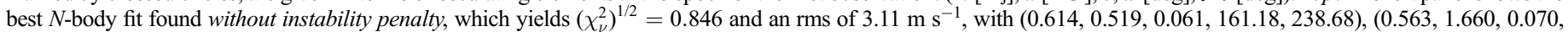
$343.97,55.17)$, and $(0.726,2.974,0.367,284.06,99.19)$ for the planets $\mathrm{b}$, c, and d, respectively, and with $V_{0}=7.536 \mathrm{~m} \mathrm{~s}{ }^{-1}$. The right panel shows a stable fit with

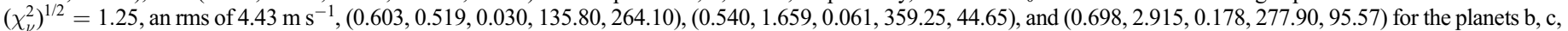

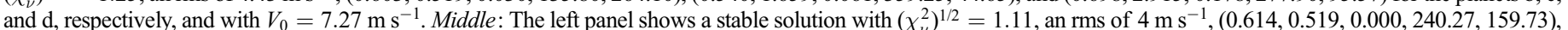

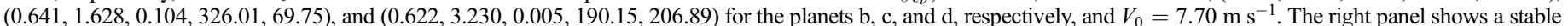

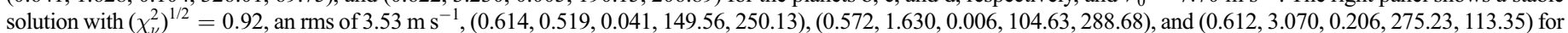

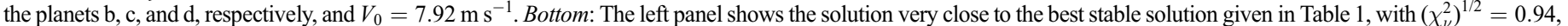

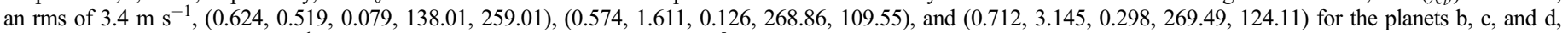

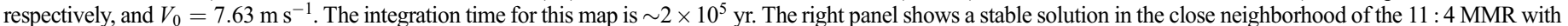

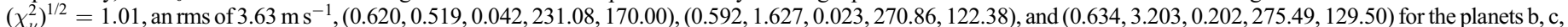

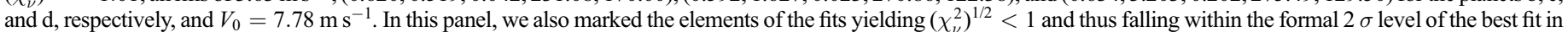

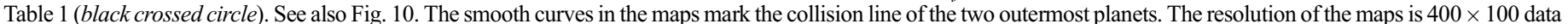
points. Integrations are for $6 \times 10^{3}$ periods of the outermost planet $\left(\sim 3 \times 10^{4} \mathrm{yr}\right)$. [See the electronic edition of the Journal for a color version of this figure.] 

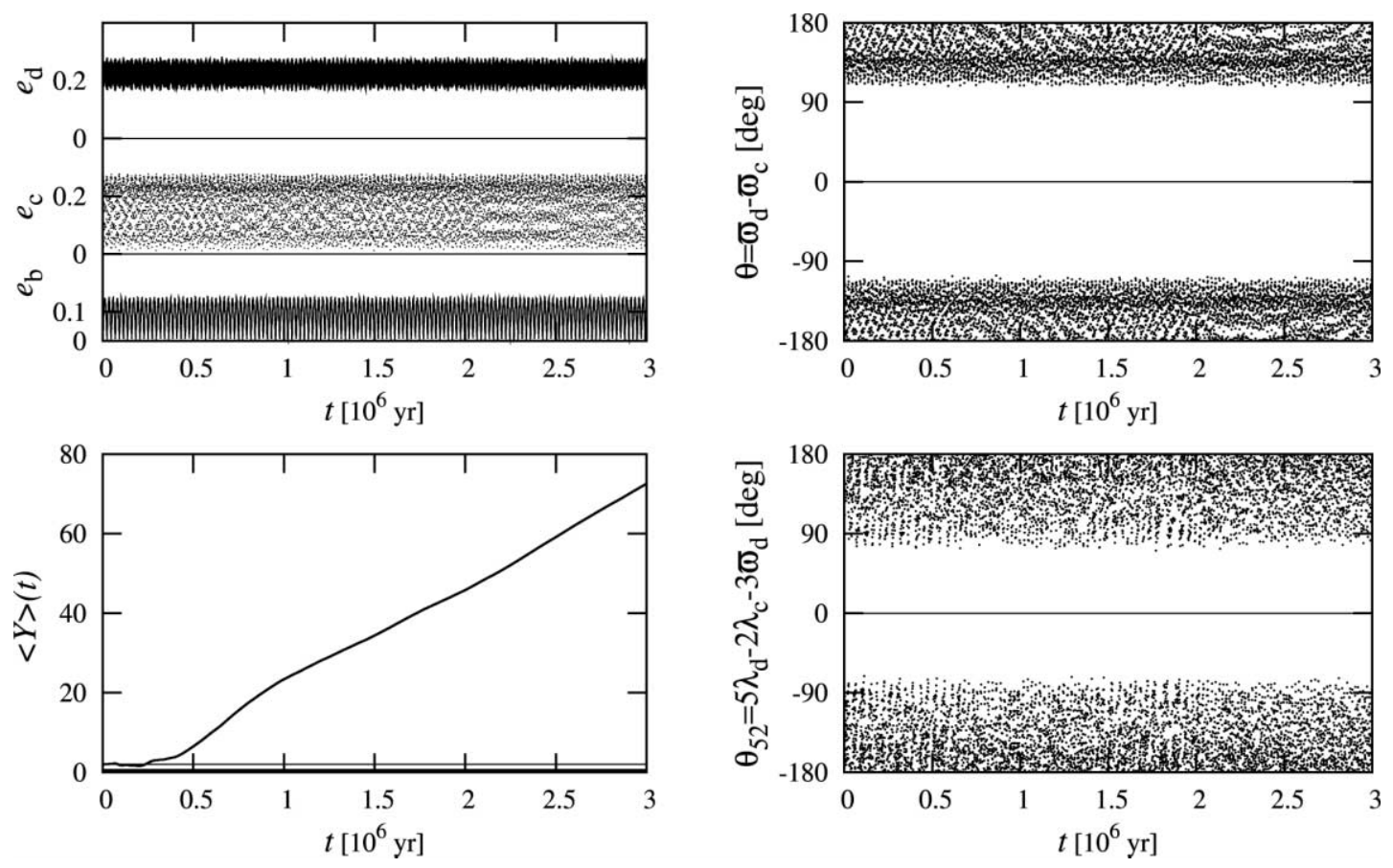

FIG. 12.-Orbital evolution of a configuration close to the best-fit solution of HD 37124 (see Table 1) and corresponding to the libration center of the 5:2 MMR. From top to bottom and left to right, we plot the eccentricities, the angle $\theta$ of the secular alignment of the apsides, the MEGNO $\langle Y\rangle$, indicating a quasi-regular configuration, and the critical argument of a 5:2 MMR. Parameters of this fit $\left[\left(\chi_{\nu}^{2}\right)^{1 / 2}=1.05\right.$, with an rms of $\left.3.82 \mathrm{~m} \mathrm{~s}^{-1}\right]$ in terms of the osculating elements at the epoch of the first observation, $\left(m\left[M_{\mathrm{J}}\right], a[\mathrm{AU}], e, \omega[\mathrm{deg}], \mathcal{M}[\mathrm{deg}]\right)$, are $(0.633,0.519,0.032,63.56,334.53)$ for planet $\mathrm{b},(0.583,1.647,0.015,124.63,281.95)$ for planet $\mathrm{c}$, and $(0.671,3.025,0.269,307.64,82.47)$ for planet $\mathrm{d}$, with $V_{0}=8.61 \mathrm{~m} \mathrm{~s}^{-1}$.

However, it remains possible (but not very likely) that we missed a better solution. Yet it could also be a dynamically derived argument against the configuration with the hot Neptune planet. Another argument against such a solution is given in a very recent work by Ford (2005), who found via the Bayesian technique that the short-period orbit (of 30 days) is not very credible.
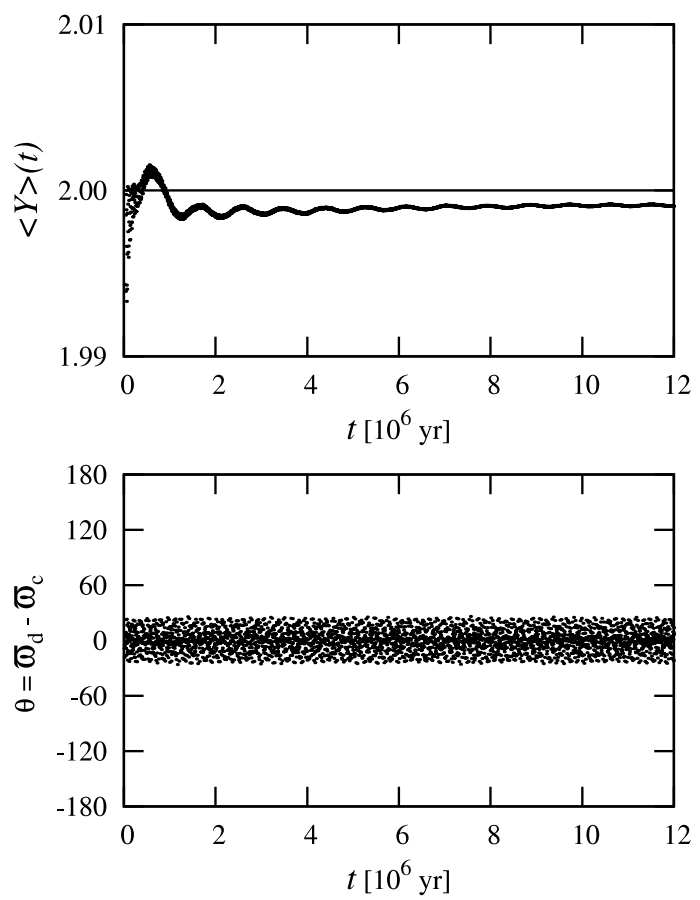

In fact, in the GA-simplex search we also found other curious triple-Keplerian solutions that were comparable to the best fits described above. The first one yields $\left(\chi_{\nu}^{2}\right)^{1 / 2}=1.029$ and an rms of $3.74 \mathrm{~m} \mathrm{~s}^{-1}$. Its parameters $\left(K, P, e, \omega, T_{p}-T_{0}\right)$ are (31.763 $\mathrm{m} \mathrm{s}^{-1}, 154.444$ days, $0.095,170.909,11557.591$ days), (13.102 $\mathrm{m} \mathrm{s}^{-1}, 318.922$ days, $0.673,50^{\circ} .785,13929.828$ days),
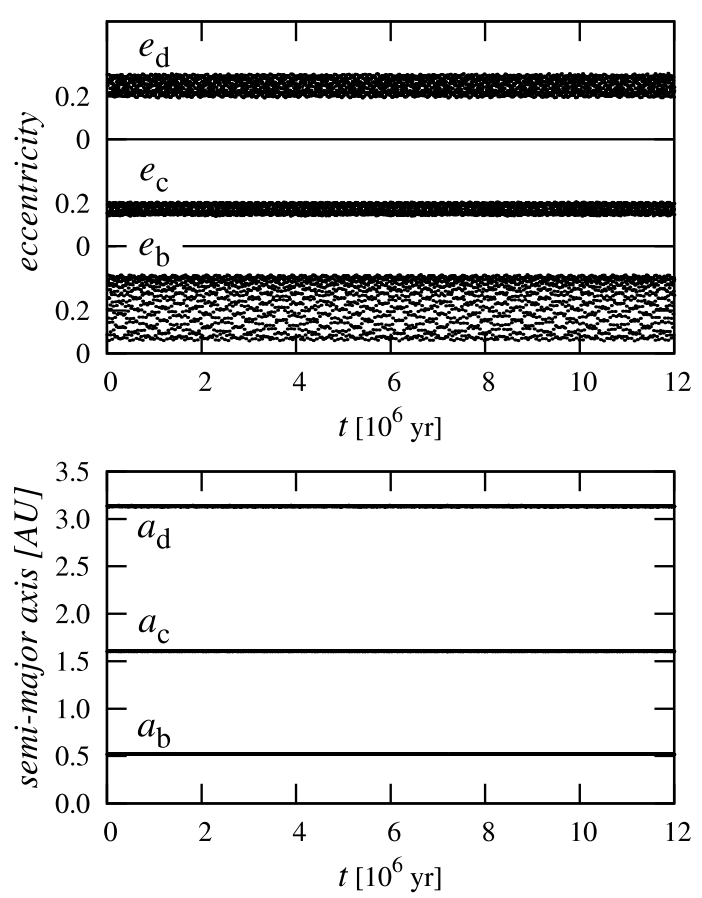

FIG. 13.- Orbital evolution of the configuration related to the best-fit solution of HD 37124 (see Table 1). From top to bottom and left to right, we plot the MEGNO $\langle Y\rangle$, the eccentricities, the angle $\theta$ of the secular alignment of the apsides of the two outermost orbits, and the semimajor axes. 


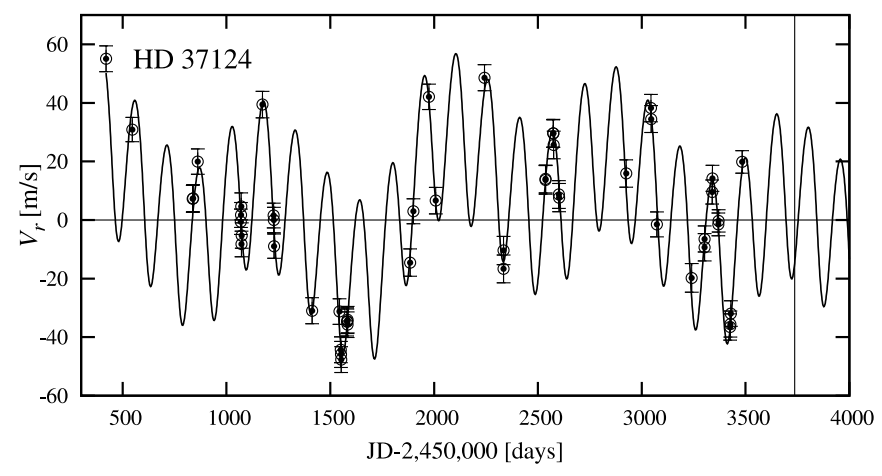

FIG. 14. - Synthetic RV curves for the best-fit solutions to the RV data of HD 37124 (see Table 1). Data points published in Vogt et al. (2005) are plotted with error bars indicating the joint RV error (stemming from the measurements and the stellar jitter). The vertical line at JD 2,453,736 indicates the calendar date 2005 December 31.

and $\left(14.828 \mathrm{~m} \mathrm{~s}^{-1}, 862.831\right.$ days, $0.379,139^{\circ} .256,11536.030$ days) for planets b, c, and d, respectively, with $T_{0}=$ JD 2, 440,000 and $V_{0}=6.229 \mathrm{~m} \mathrm{~s}^{-1}$. This solution might correspond to the $2: 1$ MMR of planets $b$ and $c$, but it appears to be very unstable (due to large $e_{b}$ and crossing orbits). An even better best-fit solution $\left[\left(\chi_{\nu}^{2}\right)^{1 / 2}=0.99\right.$, with an rms of $\left.3.66 \mathrm{~m} \mathrm{~s}^{-1}\right]$, unfortunately also unstable, is given by the following: $\left(19.794 \mathrm{~m} \mathrm{~s}^{-1}, 96.062\right.$ days, $0.789,308^{\circ} .678,13102.865$ days), (31.181 $\mathrm{m} \mathrm{s}^{-1}, 154.848$ days, $0.014,198^{\circ} .923,14361.028$ days), and $\left(16.482 \mathrm{~m} \mathrm{~s}^{-1}, 859.218\right.$ days, $0.259,214^{\circ} .801,12509.269$ days) for planets $\mathrm{b}, \mathrm{c}$, and $\mathrm{d}$, respectively, with $V_{0}=5.315$. Whether stable $N$-body solutions of these types exist is an open question that we have not investigated that closely. Yet these examples show once again that modeling of the RV data gathered for HD 37124 should not rely on the kinematic Keplerian model.

\section{IS THE GAMP NOT ALWAYS NECESSARY? (HD 108874)}

The dual planet system around HD 108874 can be close to the 4:1 MMR. That conclusion follows from the analysis of the double-Keplerian model of the RV performed by the discovery team (Vogt et al. 2005). With the HD 202206 system in mind, we suspect that the GAMP code should help us to better understand the system dynamics than follows from the kinematic approach.

According to Vogt et al. (2005), HD 108874 is an inactive star with $\log R_{\mathrm{HK}}^{\prime}=-5.07$; thus, following the discovery team, we adopted a value of $\sigma_{j}$ of $3.9 \mathrm{~m} \mathrm{~s}^{-1}$. Using the hybrid GA-simplex code, we found a slightly better two-planet Keplerian solution than the one by Vogt et al. (2005), which yielded $\left(\chi_{\nu}^{2}\right)^{1 / 2} \sim 0.79$. Our solution has $\left(\chi_{\nu}^{2}\right)^{1 / 2}=0.733$ and an rms of $3.29 \mathrm{~m} \mathrm{~s}^{-1}$. The model parameters $\left(K, P, e, \omega, T_{p}-T_{0}\right)$ are as follows: $\left(37.898 \mathrm{~m} \mathrm{~s}^{-1}\right.$, 395.276 days, $0.069,249^{\circ} .271,923.629$ days $)$ and $\left(18.355 \mathrm{~m} \mathrm{~s}^{-1}\right.$, 1600.112 days, $0.254,15^{\circ} .352,2790.983$ days) for the inner and outer planets, respectively, with $T_{0}=\mathrm{JD} 2,450,000$ and velocity offset $V_{0}=17.435 \mathrm{~m} \mathrm{~s}^{-1}$. Adopting the date of the first observation as the osculating epoch, we recalculated the inferred astrocentric osculating elements $\left(m_{p} \sin i, a, e, \omega, \mathcal{M}\right)$ as follows: $\left(1.358 M_{\mathrm{J}}, 1.046 \mathrm{AU}, 0.067,248^{\circ} .183,21^{\circ} .029\right)$ and $\left(1.015 M_{\mathrm{J}}\right.$, $\left.2.668 \mathrm{AU}, 0.254,15^{\circ} .352,33^{\circ} .733\right)$ for the inner and outer planets, respectively.

The discovery team performed dynamical simulations that relied on their double-Keplerian fit. These experiments revealed that HD 108874 is a dynamically active system. Initial conditions derived from the kinematic model may lead, depending on the initial epoch, to the destruction of the system in a timescale of about $0.5 \mathrm{Myr}$.

The results of the GAMP search are illustrated in Figure 15, which shows the solutions spanning the formal $1 \sigma, 2 \sigma$, and $3 \sigma$ confidence intervals of the best fit (its parameters are given in
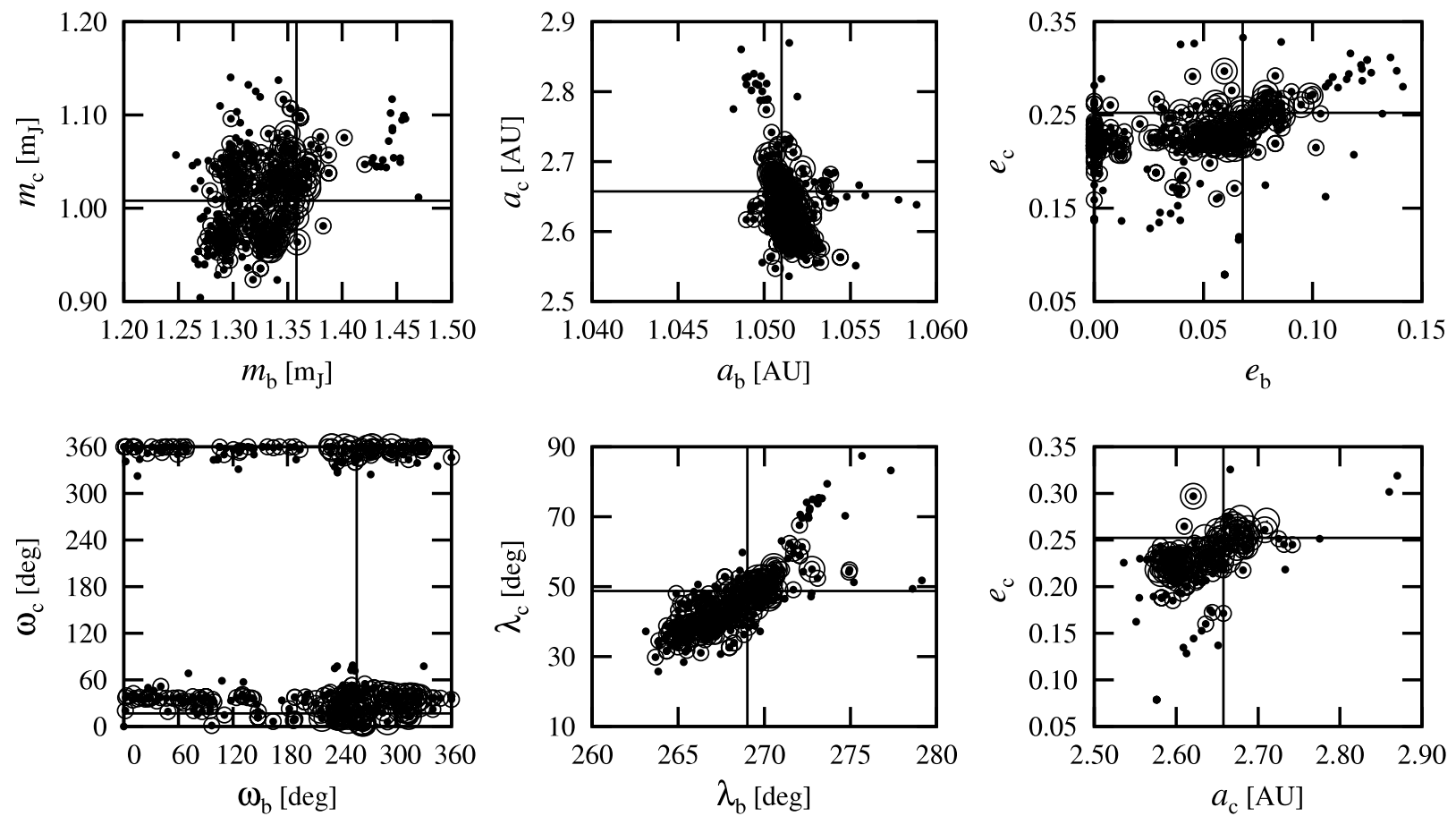

FIG. 15. - Best fits obtained with GAMP for the RV data (Vogt et al. 2005) of HD 108874. In the model, a coplanar system is assumed. Parameters of the best fits are projected onto the planes of osculating orbital elements. The smallest filled circles indicate stable solutions with $\left(\chi_{\nu}^{2}\right)^{1 / 2}<0.91$, corresponding to the $3 \sigma$ confidence interval of the best fit. Larger open circles indicate $\left(\chi_{\nu}^{2}\right)^{1 / 2}<0.82$ and $\left(\chi_{\nu}^{2}\right)^{1 / 2}<0.76$ (the $2 \sigma$ and $1 \sigma$ confidence intervals of the best-fit solution given in Table 1, respectively). The largest circles indicate the solutions with $\left(\chi_{\nu}^{2}\right)^{1 / 2}<0.713$, marginally larger than the value of $\left(\chi_{\nu}^{2}\right)^{1 / 2}=0.7126$ of the best-fit initial condition given in Table 1 . 

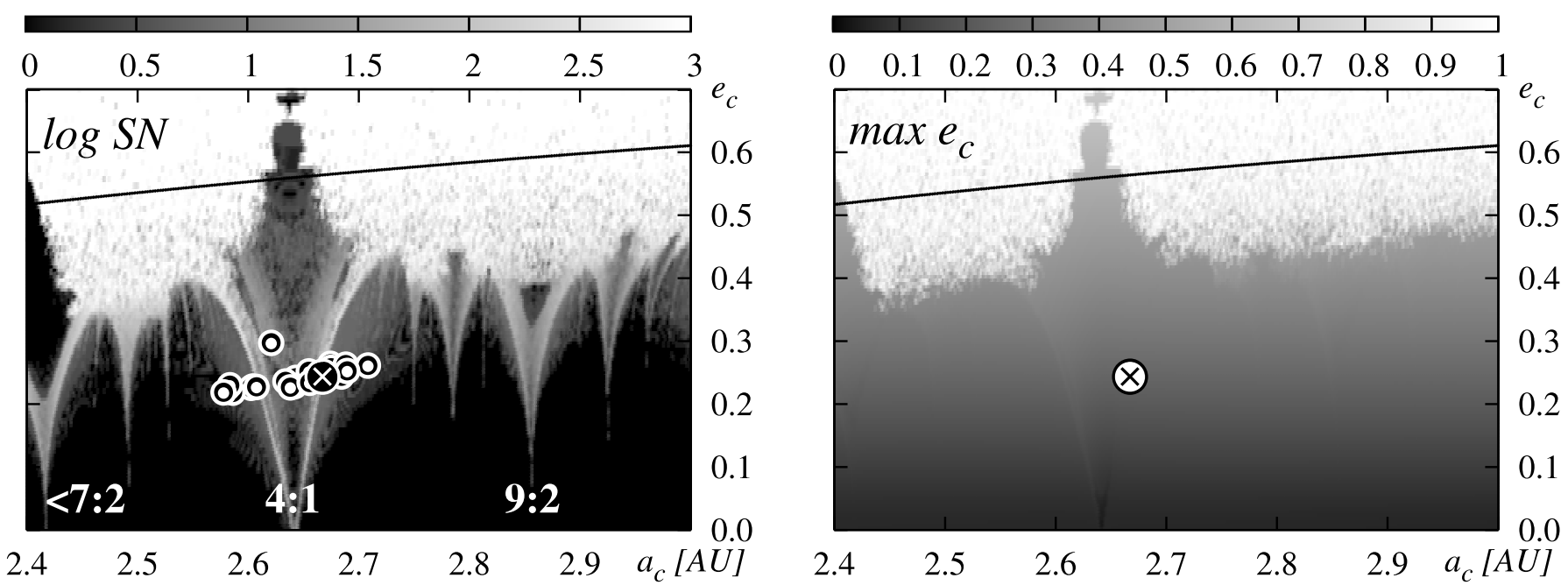

FIG. 16.-Dynamical maps in the $\left(a_{c}, e_{c}\right)$-plane in terms of the spectral number, $\log \mathrm{SN}$, and max $e_{c}$ for the best-fit solution to the HD $108874 \mathrm{RV}$ data. See Table 1 for the initial condition. Shadings used in the log SN map classify the orbits: black indicates quasi-periodic, regular configurations, while white indicates strongly chaotic systems. The resolution of the maps is $600 \times 120$ data points. Integrations are for $2 \times 10^{4}$ periods of the outer planet $\left(\sim 8.6 \times 10^{4} \mathrm{yr}\right)$. The parameters of the fits within $1 \sigma$ confidence interval of the best fit are also marked (see also Fig. 15). The crossed circle marks the initial condition used for computing the maps. [See the electronic edition of the Journal for a color version of this figure.]

Table 1). In that figure, the osculating elements of the best fits gathered by independent runs of the GAMP code are illustrated as projections onto the planes of orbital elements. This also gives us estimates of the fit errors. In the independent runs, the fits converged to the same solution as in Table 1. That solution has orbital parameters similar to those found with a double-Keplerian model of the RV; nevertheless, the quality of the fit measured by $\left(\chi_{\nu}^{2}\right)^{1 / 2} \sim 0.71$ is slightly better than that for the kinematic model.

It appears that the orbital elements of both companions are already well constrained through the available RV measurements. An interesting conclusion can be derived from the inspection of the bottom left and middle panels of Figure 15, showing the $\left(\omega_{b}\right.$, $\left.\omega_{c}\right)$-plane and the $\left(\lambda_{b}, \lambda_{c}\right)$-plane, respectively. While the values of $\omega_{b}$ of the best fits are spread over the whole possible range, both planetary longitudes are very well bounded. This means that the parameters $\omega$ and $\mathcal{M}$ (the mean anomaly) may be apparently unconstrained; nevertheless, their sum gives us a well-fixed orbital phase. Finally, we computed the dynamical maps in the $\left(a_{c}\right.$, $e_{c}$ )-plane (the left panel of Fig. 16 shows $\log \mathrm{SN}$, and the right panel shows $\max e_{c}$ ). We note that the border of the formally un-

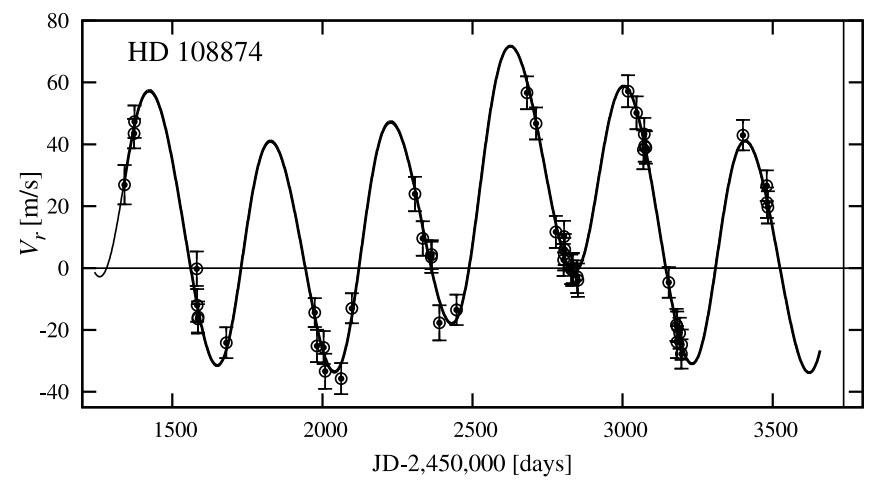

FIG. 17.-Synthetic RV curve for the best-fit solution to the RV data of HD 108874 (see Table 1; thick line). The thin line indicates the best-fit doubleKeplerian solution quoted in the text. Data points published in Vogt et al. (2005) are plotted, with error bars indicating the joint RV error (stemming from the measurements and the stellar jitter). The vertical line at JD 2,453,736 indicates the calendar date 2005 December 31. stable region begins well under the planetary collision line. However, in the libration area of the $4: 1 \mathrm{MMR}$, stable motions are possible up to $e_{c} \sim 0.7$ ! In the $\log \mathrm{SN}$ map we marked the orbital parameters of the fits within the $1 \sigma$ confidence interval of the best-fit solution. They cover the whole resonance width of about 0.05 AU. The best-fit solution is found close to the separatrix of the resonance. The synthetic RV curve is shown in Figure 17, and it perfectly follows the measurements. Finally, Figure 18 shows the orbital evolution of the configuration derived from the best fit (Table 1) and its stability analysis by MEGNO. MEGNO has been computed for over $10 \mathrm{Myr}\left(\sim 2 \times 10^{6} P_{c}\right)$ and perfectly converges to 2 at this period of time, so this configuration is strictly quasiperiodic. This is also seen in the time evolution of the eccentricities: no secular drifts are present, and their amplitudes are very small. Actually, the system is locked in the $4: 1 \mathrm{MMR}$, as the one of the critical arguments, $\theta_{41}=4 \lambda_{c}-\lambda_{b}-2 \varpi_{c}-\varpi_{b}$, librates around $0^{\circ}$.

Our conclusions are somewhat against the results of Vogt et al. (2005), who claim that the system can be currently described by a large number of dynamically distinct configurations. Curiously, the best fit found with GAMP is almost the same as the one derived without the penalty term and only marginally different ( $\sim 0.01 \mathrm{~m} \mathrm{~s}^{-1}$ in the rms) from the one obtained with the doubleKeplerian parameterization.

The results of the stability analysis carried out for the best fit (Fig. 16) suggest that in the case of HD 108874 the use of GAMP is not so critical for obtaining stable solutions as it is for other systems analyzed in this paper. It is likely due to well-constrained orbital parameters of the best fit or to a specific shape of the resonance. Its width is comparable to the fit errors. Still, without explicit computations, we cannot be sure in which region of the phase space the best fit is localized and what this region looks like.

\section{CONCLUSIONS}

With the application of GAMP, we found a clear indication of a new, second planetary companion in the 14 Her system. Remarkably, the data permit two distinct solutions corresponding to the low-order mean motion resonances $3: 1$ or $6: 1$. A few recent observations could be very useful to resolve the doubt. We also found that the two outermost planets in the HD 37124 system 

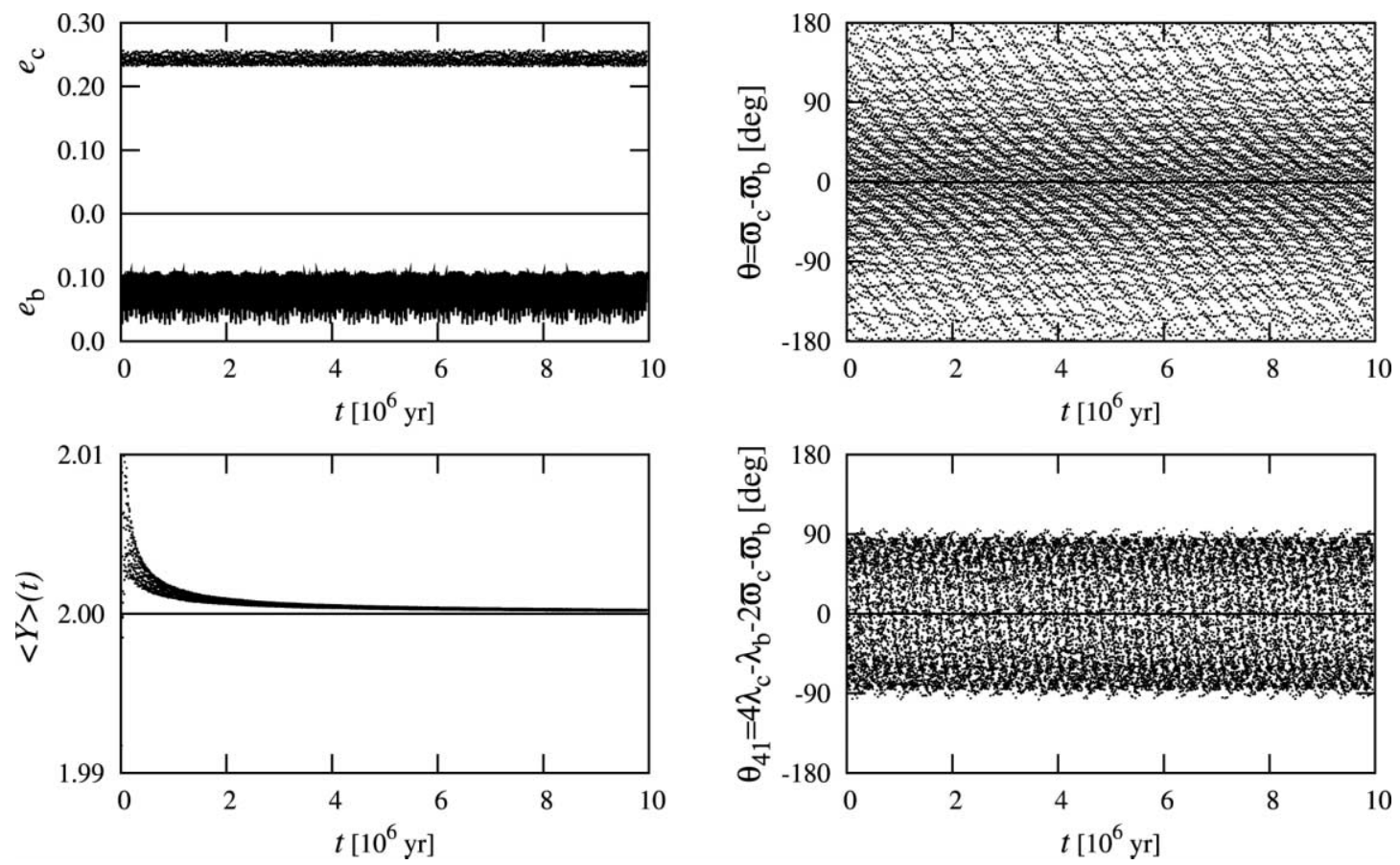

Fig. 18.- Orbital evolution of the best-fit configuration of HD 108874 (see Table 1). From top to bottom and left to right, we plot the eccentricities, the angle $\theta$ of the secular alignment of the apsides, the MEGNO $\langle Y\rangle$, and the critical argument of the $4: 1$ MMR. MEGNO is computed over $2 \times 10^{6}$ periods of the outermost planet. A perfect convergence to the value of 2 indicates a strictly quasi-regular configuration. The critical argument of the $4: 1$ MMR librates around $0^{\circ}$ : the system is locked in an exact 4:1 MMR.

may be close to a $5: 2$ MMR, thus appearing remarkably similar to the Jupiter-Saturn pair. GAMP helped us to find stable configurations of the HD 108874 system that support the hypothesis that the system is locked in an exact $4: 1$ MMR.

As a by-product of our analysis, we have provided new examples that confirm good properties of the GAMP algorithm. Indeed, the idea has a solid theoretical background. By applying the obvious requirement of the dynamical stability, we eliminate the initial conditions that lead to a quick destruction of a planetary configuration. A delicate matter is the question of how to understand (and measure) the stability. In this paper we prefer the formal definition provided by the KAM theorem. Essentially, the dynamics of a planetary system has two timescales related to the fast orbital motions and their resonances (MMRs) and the much slower precession of instantaneous orbits (secular dynamics). In analyzing the relatively small sets of the RV measurements and due to narrow observational windows, we are naturally limited to the short timescale. The recent secular theories by Michtchenko \& Malhotra (2004) and Lee \& Peale (2003) for two-planet systems and the results of Pauwels (1983) for a general $N$-planet system in the regime of moderate eccentricities are very useful for predicting the generic features of such systems. They are generically stable under the condition that planets are not involved in strongly chaotic motions (usually related to loworder MMRs) or that their orbits stay far from collision zones. Our line of reasoning is that, at least in the first approximation, we should eliminate initial conditions corresponding to such unstable behaviors. This is possible thanks to computationally efficient fast indicators. Yet, according to the KAM theorem, the search for the best-fit solutions is conducted in a highly noncontinuous parameter space. A cure for this problem is an application of nongradient genetic algorithms that have features ideally suited to our purposes. The GAs need only to "know" the $\left(\chi_{\nu}^{2}\right)^{1 / 2}$ function to efficiently explore the phase space. To eliminate the unstable solutions we add a penalty term to the formal $\left(\chi_{\nu}^{2}\right)^{1 / 2}$ of potential solutions. Let us underline that such a penalty term may be arbitrary, so in fact we may use virtually any criterion of stability. Still, one should be aware that the GAMP-like code is CPUexpensive. For instance, the GAMP calculations typically take several days of a 16 processor AMD Opteron $2 \mathrm{GHz}$ cluster for every system studied in this paper. Nevertheless, the method may be optimized in many ways.

The multiplanet configurations analyzed in this paper are representative cases in which we may benefit from the application of GAMP-like code. Frequently, the RV data span a short time with respect to the longest orbital periods, and then pure Keplerian, or even $\mathrm{N}$-body Newtonian, models of the reflex motion of the parent star yield physically unacceptable configurations that disrupt during thousands of years. That obviously contradicts the Copernican principle. A good example of such a situation is provided by the $\mu$ Ara system (Jones et al. 2002; McCarthy et al. 2004; Goździewski et al. 2005) or the 14 Her system (Butler et al. 2003; Naef et al. 2004) analyzed in this paper. In both instances, the RV data indicate linear trends over the RV signal of a single-planet configuration. In such instances, the GAMP-like code makes it possible to limit significantly the otherwise completely unconstrained parameters of the putative long-period companions.

It is important to stress that a GAMP-like code (with any kind of a instability penalty) by no means modifies the $N$-body model that is used as an internal representation of the planetary system. It does not increase the number of fitted parameters. We also do not assume ad hoc that only one "excellent" (for instance, strictly resonant) best-fit solution exists. Using the GAMP-like approach, we are able to simultaneously analyze many local minima of $\left(\chi_{\nu}^{2}\right)^{1 / 2}$. The code enables us to illustrate the space of stable multiparameter best-fit solutions within prescribed error bounds as projections of their elements onto chosen parameter planes. Indeed, the case of 14 Her shows that there exist two equally deep and well-defined local $\left(\chi_{\nu}^{2}\right)^{1 / 2}$ minima that are far from each other in the parameter space. Another example is the 
HD 37124 system, for which we found many qualitatively different configurations and resolved the system's complex phase space. Yet another example is the HD 128311 system, which we analyzed in our recent paper (Goździewski \& Konacki 2006). In that case, the RV curve can be described equally well by two completely different $2: 1$ and $1: 1$ MMR configurations.

The GAMP-like algorithm is especially well suited for the analysis of RV data of stars hosting multiplanet systems with Jovian planets likely involved in strong, low-order MMRs. Such systems are naturally favored by the Doppler technique because of their relatively short observational windows. We have illustrated the efficiency of the method by analyzing the measurements of HD 202206 (Correia et al. 2005), HD 37124 and HD 108874 (Vogt et al. 2005), HD 128311 (Vogt et al. 2005), and HD 82943 (Mayor et al. 2004), which we also studied in our recent paper (Goździewski \& Konacki 2006). In all these cases, the stability zones are very sharp and the formal (KAM-like) and astronomical notions of stability are strictly related to each other. Then it is essential to use the stability criterion as an internal part of the fitting algorithm. The GAMP-like code makes it possible to find the statistically optimal, stable solutions. The stability analysis is also greatly simplified. Certainly the dynamical analysis of other resonant systems may also benefit from the application of this numerical tool.

According to Marcy et al. (2005), nearly all giant planets orbiting within $2 \mathrm{AU}$ of all F, G, and K stars within $30 \mathrm{pc}$ have now been discovered. The observational windows of the extrasolar searches are constantly widening. The orbital periods of newly revealed candidate planets become longer and longer. The full coverage of their periods by observations has already become a matter of many years. In this context, the GAMP analysis may be useful to significantly constrain the orbital elements of long-period planetary companions and to plan the optimal observational strategy.

We kindly thank Zbroja for correcting our manuscript. This work is supported by the Polish Ministry of Science and Education grant 1P03D 02129 . M. K. is also supported by NASA through grant NNG04GM62G. K. G. is also supported by N. Copernicus Univ. through grant 427A.
Adams, F. C., \& Laughlin, G. 2003, Icarus, 163, 290

Arnold, V. I. 1978, Mathematical Methods of Classical Mechanics (New York: Springer)

Butler, R. P., Marcy, G. W., Vogt, S. S., Fischer, D. A., Henry, G. W., Laughlin, G., \& Wright, J. T. 2003, ApJ, 582, 455

Charbonneau, P. 1995, ApJS, 101, 309

Cincotta, P. M., Giordano, C. M., \& Simó, C. 2003, Physica D, 182, 151

Cincotta, P. M., \& Simó, C. 2000, A\&AS, 147, 205

Correia, A. C. M., Udry, S., Mayor, M., Laskar, J., Naef, D., Pepe, F., Queloz, D., \& Santos, N. C. 2005, A\&A, 440, 751

Ferraz-Mello, S., Michtchenko, T. A., \& Beaugé, C. 2005, ApJ, 621, 473

Fischer, D. A., et al. 2003, ApJ, 586, 1394

Ford, E. B. 2005, in Protostars and Planets V, ed. V. Mannings et al. (Houston: Lunar Planet. Inst.), 8358

Giordano, C. M., \& Cincotta, P. M. 2004, A\&A, 423, 745

Goździewski, K. 2003, A\&A, 398, 315

Goździewski, K., \& Konacki, M. 2006, ApJ, in press (astro-ph/0510109)

Goździewski, K., Konacki, M., \& Maciejewski, A. J. 2003, ApJ, 594, 1019

. 2005, ApJ, 622, 1136

Goździewski, K., \& Maciejewski, A. J. 2001, ApJ, 563, L81

. 2003, ApJ, 586, L153

Goździewski, K., \& Migaszewski, C. 2006 A\&A, 449, 1219

Jones, H. R. A., Butler, R. P., Marcy, G. W., Tinney, C. G., Penny, A. J., McCarthy, C., \& Carter, B. D. 2002, MNRAS, 337, 1170

Laskar, J. 1993, Celest. Mech. Dyn. Astron., 56, 191

Laughlin, G., \& Chambers, J. E. 2001, ApJ, 551, L109

\section{REFERENCES}

Lecar, M., Franklin, F. A., Holman, M. J., \& Murray, N. J. 2001, ARA\&A, 39, 581

Lee, M. H., \& Peale, S. J. 2003, ApJ, 592, 1201

Lissauer, J. J. 1999, Rev. Mod. Phys., 71, 835

Marcy, G., Butler, R. P., Fischer, D., Vogt, S., Wright, J. T., Tinney, C. G., \& Jones, H. R. A. 2005, Prog. Theor. Phys. Suppl., 158, 24

Mayor, M., Udry, S., Naef, D., Pepe, F., Queloz, D., Santos, N. C., \& Burnet, M. 2004, A\&A, 415, 391

McCarthy, C., Butler, R. P., Tinney, C. G., Jones, H. R. A., Marcy, G. W., Carter, B., Penny, A. J., \& Fischer, D. A. 2004, ApJ, 617, 575

Michtchenko, T. A., \& Ferraz-Mello, S. 2001, AJ, 122, 474

Michtchenko, T. A., \& Malhotra, R. 2004, Icarus, 168, 237

Murray, C. D., \& Dermott, S. F. 2000, Solar System Dynamics (Cambridge: Cambridge Univ. Press)

Naef, D., Mayor, M., Beuzit, J. L., Perrier, C., Queloz, D., Sivan, J. P., \& Udry, S. 2004, A\&A, 414, 351

Pauwels, T. 1983, Celest. Mech., 30, 229

Press, W. H., Teukolsky, S. A., Vetterling, W. T., \& Flannery, B. P. 1992 Numerical Recipes in C (2nd ed.; Cambridge: Cambridge Univ. Press)

Thommes, E. W., \& Lissauer, J. J. 2003, ApJ, 597, 566

Udry, S., Mayor, M., Naef, D., Pepe, F., Queloz, D., Santos, N. C., \& Burnet, M. 2002, A\&A, 390, 267

Vogt, S. S., Butler, R. P. Marcy, G. W., Fischer, D. A., Henry, G. W., Laughlin, G., Wright, J. T., \& Johnson, J. A. 2005, ApJ, 632, 638

Wright, J. T. 2005, PASP, 117, 657 